\title{
Bronchopulmonary dysplasia
}

Bernard Thébaud ${ }^{1,2 *}$, Kara N. Goss ${ }^{3}$, Matthew Laughon $^{4}$, Jeffrey A. Whitsett ${ }^{5}$, Steven H. Abman ${ }^{6}$, Robin H. Steinhorn ${ }^{7}$, Judy L. Aschner ${ }^{8,9}$, Peter G. Davis ${ }^{10}$, Sharon A. McGrath-Morrow ${ }^{11}$, Roger F. Soll ${ }^{12}$ and Alan H. Jobe

Abstract | In the absence of effective interventions to prevent preterm births, improved survival of infants who are born at the biological limits of viability has relied on advances in perinatal care over the past 50 years. Except for extremely preterm infants with suboptimal perinatal care or major antenatal events that cause severe respiratory failure at birth, most extremely preterm infants now survive, but they often develop chronic lung dysfunction termed bronchopulmonary dysplasia (BPD; also known as chronic lung disease). Despite major efforts to minimize injurious but often life-saving postnatal interventions (such as oxygen, mechanical ventilation and corticosteroids), BPD remains the most frequent complication of extreme preterm birth. BPD is now recognized as the result of an aberrant reparative response to both antenatal injury and repetitive postnatal injury to the developing lungs. Consequently, lung development is markedly impaired, which leads to persistent airway and pulmonary vascular disease that can affect adult lung function. Greater insights into the pathobiology of BPD will provide a better understanding of disease mechanisms and lung repair and regeneration, which will enable the discovery of novel therapeutic targets. In parallel, clinical and translational studies that improve the classification of disease phenotypes and enable early identification of at-risk preterm infants should improve trial design and individualized care to enhance outcomes in preterm infants.

In 1967, Northway, Rosen and Porter described a new lung disease in preterm infants who had hyaline membrane disease (now known as respiratory distress syndrome (RDS; see BOX 1 for a description of neonatal terms), which at that time was a highly lethal condition that resulted from using mechanical ventilation without positive end-expiratory pressure and high levels of supplemental oxygen in an attempt to save these infants ${ }^{1}$. In that era, survival was rare despite a relatively mild degree of prematurity (mean gestational age, 34 weeks), but many preterm infants had a slow and prolonged recovery from airway and lung parenchymal injury. The authors termed this new syndrome bronchopulmonary dysplasia (BPD; also known as chronic lung disease) on the basis of airway histopathological features.

The characteristics of BPD have evolved over the past 50 years, as most preterm infants now survive with the use of antenatal corticosteroids, advanced neonatal care techniques, effective respiratory support devices and surfactant treatments ${ }^{2}$. A current perspective regarding the initiation, progression and variable extent of lung repair of the most extremely preterm infants who are at highest risk of $\mathrm{BPD}^{3}$ is shown in FIG. 1. In the framework of the Developmental Origins of Adult Diseases concept, transgenerational and pre-conception factors interact with pregnancy exposures to modulate the risk of BPD, and gestational age at birth is the greatest single predictor of $\mathrm{BPD}$. It is essential to recognize that no infant born very preterm is 'normal', because of many factors, including abnormalities of pregnancy that result in preterm birth, and the fact that health problems in a preterm newborn are more severe the earlier the birth ${ }^{4}$. The remarkable plasticity of the lung development programme to support very early gestational lung maturation is crucial for the survival of extremely-low-birth-weight (ELBW; $<1,000 \mathrm{~g}$ ) infants. However, the structurally very immature lungs of these infants are easily injured by the routine care required to ensure their survival. Remarkably, lung development can proceed after preterm birth despite ongoing lung injury from supplemental oxygen and mechanical ventilation and/or lung stretch. BPD is the clinical expression of this mixture of lung developmental plasticity, injury and repair that ultimately results in a remarkable ability of the lungs of surviving infants to remodel until perhaps 20 years of age ${ }^{5}$.

In this Primer, we discuss the different definitions of BPD, ongoing controversy about the optimal time point and method for determining whether BPD exists, and how this variability might affect assessment of disease prevalence. Furthermore, we review advances in neonatal care, such as non-invasive ventilation and alternative strategies for surfactant administration, which 


\author{
Author addresses \\ ${ }^{1}$ Division of Neonatology, Department of Pediatrics, Children's Hospital of Eastern \\ Ontario (CHEO) and CHEO Research Institute, Ottawa, Ontario, Canada. \\ ${ }^{2}$ Ottawa Hospital Research Institute, Sinclair Centre for Regenerative Medicine, Ottawa, \\ Ontario, Canada. \\ ${ }^{3}$ Departments of Medicine and Pediatrics, University of Wisconsin-Madison, Madison, \\ WI, USA. \\ ${ }^{4}$ The University of North Carolina at Chapel Hill, Chapel Hill, NC, USA. \\ ${ }^{5}$ Cincinnati Children's Hospital Medical Center, Division of Neonatology, Pulmonary \\ Biology, University of Cincinnati, Cincinnati, OH, USA. \\ ${ }^{6}$ Pediatric Heart Lung Center, Section of Pulmonary Medicine, Department of Pediatrics, \\ Children's Hospital Colorado and the University of Colorado Denver Anschutz Medical \\ Center, Aurora, CO, USA. \\ ${ }^{7}$ Children's National Medical Center, Department of Pediatrics, Washington, DC, USA. \\ ${ }^{8}$ Joseph M. Sanzari Children's Hospital, Hackensack Meridian Health School of Medicine, \\ Hackensack, NJ, USA. \\ ${ }^{9}$ Albert Einstein College of Medicine, Bronx, NY, USA. \\ ${ }^{10}$ The Royal Women's Hospital, University of Melbourne, Melbourne, Victoria, Australia. \\ ${ }^{11}$ Department of Pediatrics, Eudowood Division of Respiratory Sciences, Johns Hopkins \\ University School of Medicine, Baltimore, MD, USA. \\ ${ }^{12}$ Department of Pediatrics, Larner School of Medicine, University of Vermont, Burlington, \\ VT, USA.
}

are improving outcomes in preterm infants with BPD. Last, we examine various key issues that require further research to improve our understanding of disease pathobiology and enable development of better interventions that may enhance short-term and long-term outcomes.

\section{Epidemiology Prevalence}

Premature birth ( $<37$ weeks gestational age) is common and affects $6-14 \%$ of pregnancies, depending on the country ${ }^{6,7}$. Generally, premature infants are classified on the basis of gestational age (extremely preterm infants or extremely low gestational age newborns (ELGANs) are $<28$ weeks gestation; very preterm infants are 28 to $<32$ weeks gestation, moderate preterm infants are 32 to $<34$ weeks, and late preterm infants are 34 to $<37$ weeks) or birth weight (ELBW infants are $<1,000 \mathrm{~g}$, verylow-birth-weight (VLBW) infants are $<1,500 \mathrm{~g}$, and lowbirth-weight (LBW) infants are $<2,500 \mathrm{~g}$ ). BPD incidence increases as gestational age and weight at birth decreases. $\mathrm{BPD}$ remains the most common complication associated with prematurity and is increasing in prevalence, most likely due to the increased survival of ELGANs ${ }^{8,9}$. Almost 50,000 ELGANs are born each year in the USA, and $\sim 35 \%(18,000)$ of these children develop BPD. The incidence of BPD varies widely between centres $(\sim 20-75 \%)$, even after adjusting for potential risk factors $^{10}$. Data from major cohort studies (such as ELGAN, Canadian Neonatal Network, Korean Neonatal Network, Vermont-Oxford Network and Swiss Neonatal Network, as well as studies in China, Taiwan and India) demonstrate a BPD prevalence of $11-50 \%$, a wide range that is due to differences in gestational age or birth weight criteria for a BPD diagnosis ${ }^{11-17}$.

Marked variations in neonatal outcomes between countries have been identified in a large, multicentre, multinational cohort of 58,004 very preterm and VLBW infants from Australia, New Zealand, Canada, Israel, Japan, Spain, Sweden, Switzerland and the UK ${ }^{18}$. For example, Japan had the lowest mortality but the highest rate of treatment for retinopathy of prematurity, whereas Spain had the highest mortality but a comparatively lower prevalence of BPD. These variations could be the result of differences in population coverage, organization of perinatal health-care delivery, population characteristics, case definitions, ascertainment, data quality and reliability, and care processes, and highlight the need for international multicentre clinical trials and international benchmarking and quality improvement activities.

\section{Risk factors}

The strongest risk factors for BPD are prematurity and low birth weight ${ }^{19-25}$. Almost $80 \%$ of infants who are born at 22-24 weeks of gestation are diagnosed with $\mathrm{BPD}^{26}$, whereas only $20 \%$ of infants born at 28 weeks of gestation develop BPD. Among infants with BPD, 95\% are $\mathrm{VLBW}^{27}$. Other perinatal risk factors include intrauterine growth restriction (IUGR) ${ }^{13}$, male $\operatorname{sex}^{13,20,23}$ and, inconsistently, chorioamnionitis ${ }^{28}$, race or ethnicity ${ }^{13,20,23}$, and smoking ${ }^{29,30}$. Genetic risk factors may also contribute to the development of BPD, as indicated by twin studies $^{31,32}$, and there is an ongoing search for genetic markers for $\mathrm{BPD}^{33-37}$.

Early respiratory patterns of premature infants provide insight into risk factors for BPD. An early study suggested that peak inspiratory ventilator pressure and requirement for assisted ventilation on day 4 of life are early predictors of $\mathrm{BPD}^{38}$. Subsequent studies found that three patterns of lung disease generally emerge in the first 2 weeks of life ${ }^{39-45}$ (FIG. 2). In the first pattern, infants have fairly minimal lung disease and progressively recover. In the second pattern, early persistent pulmonary deterioration (EPPD), substantial and prolonged respiratory support is required from birth. In the third pattern, an initial improvement in lung disease in the first week of life is followed by a respiratory decompensation termed pulmonary deterioration, which often requires mechanical ventilation and an increase in supplemental oxygen. Risk factors that may be associated with pulmonary deterioration include late surfactant deficiency ${ }^{46}$, sepsis, increased levels of inflammatory proteins (such as RANTES $)^{47}$ and patent ductus arteriosus ${ }^{40,43}$. Almost $50 \%$ of infants with pulmonary deterioration and almost $70 \%$ of infants with EPPD develop BPD ${ }^{48}$.

The National Institute of Child Health and Human Development (NICHD) Neonatal Research Network developed an online, publicly available risk estimator (https://neonatal.rti.org/index.cfm) that accurately estimates the risk of developing BPD by postnatal day ${ }^{49}$. Investigators identified risk factors for BPD, and the competing outcome of death, among gestational age, birth weight, ethnicity and sex, ventilatory support (ranging from no support (breathing room air without positive airway pressure) to high frequency ventilation) and fraction of inspired oxygen $\left(\mathrm{FiO}_{2}\right)$, on postnatal days $1,3,7,14$, 21 and 28 in 3,636 infants born at 23-30 weeks of gestation. The BPD prediction tool is internally and externally validated. The models predict the correct level of BPD or the occurrence of death in $>80 \%$ of cases and have the highest area under the curve (AUC) among current BPD risk predictors ${ }^{50}$. This tool is used to provide counselling to families and to quantify risk for determining patient 
inclusion in early phase therapeutic trials. Interestingly, systemic inflammation occurs early in the neonatal period and precedes clinical symptoms in infants with $\mathrm{BPD}^{51}$. This finding suggests that a therapeutic window of opportunity exists during the early phase of BPD.

\section{Mechanisms/pathophysiology Lung development and surfactant}

Normal lung development. Respiration after birth is entirely dependent on the architecture of the peripheral lung saccules and alveoli. The close interface between alveolar epithelial cells and endothelial cells of the pulmonary microvasculature enables efficient exchange of oxygen and carbon dioxide (FIG. 3). Lung formation begins

\section{Box 1 Neonatal definitions and intensive care interventions}

\begin{abstract}
Definitions
Prematurity: Birth before 37 completed weeks of gestation. This is further subdivided on the basis of gestational age into extremely preterm ( $<28$ weeks of gestation), very preterm (28-32 weeks of gestation) and moderate or late preterm (32-37 weeks of gestation).
\end{abstract}

Very low birth weight (VLBW): Infants who weigh $<1,500 \mathrm{~g}$ at birth. Most VLBW infants are born at $<30$ weeks gestational age.

Extremely low birth weight (ELBW): Infants who weigh $<1,000 \mathrm{~g}$ at birth. Most ELBW infants are born at $<28$ weeks gestational age.

Extremely low gestational age newborn: Infants born at $<28$ weeks gestational age.

Post-menstrual age: A measure of the age of an infant that combines gestational and postnatal age, both in weeks. For example, a 23-week gestational age infant at 9 weeks after birth has a postmenstrual age of 32 weeks.

Hyaline membrane disease: Also known as respiratory distress syndrome, this is the formation of a characteristic translucent membrane in collapsed alveoli, which can result from inadequate pulmonary surfactant production and structural immaturity in the under-developed lungs of preterm infants.

Intrauterine growth restriction (IUGR): Also known as fetal growth restriction, IUGR usually refers to infants whose weight is below the tenth percentile for infants of that gestational age or less than two standard deviations below the average weight for infants of that gestational age.

Persistent pulmonary hypertension of the newborn: Sustained elevation of pulmonary vascular resistance after birth that can cause profound hypoxaemia due to extrapulmonary right-to-left shunting across the foramen ovale and/or ductus arteriosus.

\section{Interventions}

Conventional mechanical ventilation: Positive airway pressure through an endotracheal tube. There are multiple ways to provide ventilation. Examples include time cycled, pressure limited; volume targeted; and neurally adjusted ventilatory assist.

Fraction of inspired oxygen $\left(\mathrm{FiO}_{2}\right)$ : The percentage of oxygen content that is involved in gas exchange in the alveoli. Supplemental oxygen usually has an $\mathrm{FiO}_{2}$ of $<0.5$ to avoid oxygen toxicity.

High-frequency jet ventilation: Ventilation through an endotracheal tube, usually with a device that is paired with a conventional mechanical ventilator and provides high rates around a targeted mean airway pressure with active inhalation and passive exhalation.

High-frequency oscillatory ventilation: Ventilation through an endotracheal tube, usually with a device that provides high rates around a mean airway pressure, with active inspiration and expiration.

Nasal continuous positive airway pressure: Continuous distending pressure, usually measured in centimetres of water, through the nasal passage (with a nasal or face mask instead of an endotracheal tube), with blended $\mathrm{FiO}_{2}$.

Nasal intermittent positive pressure ventilation: Usually a baseline continuous distending pressure with intermittent higher levels of pressure, which can be synchronized or non-synchronized, with blended $\mathrm{FiO}_{2}$.

Inhaled nitric oxide: A selective pulmonary vasodilator that reverses pulmonary vasoconstriction by modulation of vascular muscle tone. between week 3 and week 6 of gestation. Subsets of endodermal cells from the anterior foregut form the initial tracheal and pulmonary lung buds that invade the splanchnic mesenchyme. Branching morphogenesis ${ }^{52}$ forms the conducting airways and peripheral acinar buds that will ultimately form the alveoli after birth (FIG. 4). Complex paracrine interactions among various mesenchymal cells, including pericytes, smooth muscle cells, endothelial cells and various types of fibroblasts, and respiratory epithelial cells and endothelial progenitor cells are involved in the formation of the pulmonary parenchyma ${ }^{53-55}$. Acinar structures dilate later in gestation, creating the peripheral saccules (the primitive gas exchange portion of the lung) and alveoli that will accommodate postnatal adaptation to breathing. During morphogenesis, the lung is transformed from a solid gland-like tissue to an expanded open alveolar structure in which gas exchange can occur (FIG. 5). During the last trimester (28-40 weeks of gestation), peripheral saccules undergo a process of septation that further divides the airspaces, creating and increasing the number of alveoli and thereby increasing the surface area for gas exchange. Peripheral lung epithelial cells mature into alveolar type 1 (AT1) cells and AT2 cells, the latter producing pulmonary surfactant (FIG. 3). Thus, preterm infants at the edge of viability (22-23 weeks of gestation) begin ventilation at the canalicular-saccular stage of lung formation, well before morphogenesis and alveolar differentiation are completed.

Pulmonary surfactant. Pulmonary surfactant is a complex mixture of phospholipids, primarily phosphatidylcholine, and the surfactant proteins SP-A, SP-B, SP-C and SP-D, which together define the physical structure, function and metabolism of surfactant in the alveolus (FIG. 3). Synthesis of surfactant lipids and proteins depends on the differentiation of AT2 cells, which occurs fairly late in gestation. Consequently, a lack of pulmonary surfactant as a result of incomplete differentiation of AT2 cells causes RDS in preterm infants ${ }^{56}$. Assisted ventilation, continuous airway pressure and supplemental oxygen can initially support ventilation after birth. Furthermore, administration of exogenous pulmonary surfactant has greatly facilitated the transition to air breathing, decreasing the requirement for oxygen and assisted ventilation and thereby improving survival but secondarily increasing the number of infants at risk of BPD.

In conclusion, postnatal adaptation of the preterm lungs to breathing at birth is challenged by initial lung injury owing to surfactant deficiency, exposure to increased oxygen, mechanical ventilation, inadequate nutrition, infection and inflammation, which together provide considerable hurdles to normal pulmonary growth and repair and result in a loss of alveolar surface area (FIG. 5) that has lifelong consequences.

\section{Pathophysiology of acute lung injury}

BPD is a clinical syndrome of lung injury that disrupts alveolarization and microvascular development. The anatomical changes in BPD result in abnormal gas exchange and lung mechanics. However, of note, the pathological features of BPD are based on only a few 


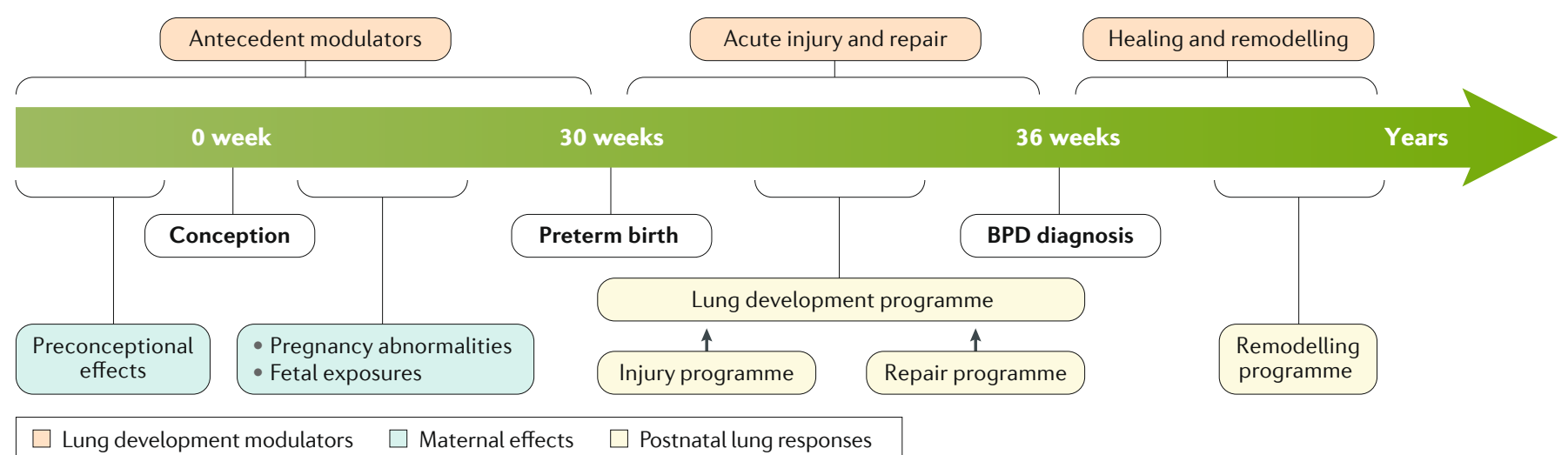

Fig. 1 | Timeline and stages of BPD. The timeline indicates variables that may modulate lung development from preconception through fetal development before preterm birth. Acute injury (on the timescale of days and weeks) resulting from neonatal care that is required to ensure survival then progresses to chronic lung injury and, ultimately, repair and remodelling over months and years. The unique aspect of bronchopulmonary dysplasia (BPD) is that it is an injury process that occurs as the premature lung is being injured and must repair as the lung continues to develop and mature. Remodelling of the lungs can occur over years.

reports that primarily describe necropsy specimens ${ }^{57}$. In fact, most patients with BPD survive, and imaging studies reveal that they probably have a very heterogeneous pathology throughout the lungs, including regions of decreased alveolarization, cystic emphysema, fibrosis and variable airway injury ${ }^{58}$. This complex injury results primarily from damage to the very preterm lungs by multiple antenatal and postnatal exposures, each of which may disrupt specific developmental pathways and promote injury (FIG. 1).

An extensive review of publications over an 18 month period (January 2015 to June 2017) identified 60 genes in transgenic mouse models that result in altered mouse lung development with characteristics of $\mathrm{BPD}^{59}$. Inflammation is the common pathway that leads to a BPD phenotype. Supplemental oxygen, positive pressure ventilation and postnatal sepsis, which all cause lung inflammation, are clinically associated with BPD. In animal models, supplemental oxygen alone or positive pressure ventilation alone cause a BPD phenotype (that is, decreased alveolarization and microvascular injury $)^{60}$. The most frequently used animal models are term mice or rats that are exposed to supplemental oxygen or preterm sheep or primates that are injured by excessive oxygen and/or ventilation or stretch ${ }^{61}$. Numerous studies have shown that blockers of inflammatory mediators, receptors and signalling pathways improve the BPD phenotype in mice, rats and rabbits ${ }^{59}$.

Unfortunately, proinflammatory cascades are complex and redundant. Although inflammatory responses in preterm lungs may differ from those in term or adult lungs ${ }^{62}$, limited clinical attempts to block oxidants with antioxidants and to block inflammation with inflammatory modulators have been uniformly unsuccessful in treating BPD in infants ${ }^{63,64}$, with the exception of postnatal corticosteroids. Corticosteroids decrease the incidence of BPD when given soon after birth and prevent the progression of lung injury to BPD when given in the first weeks of life ${ }^{65}$. For example, the potent general antiinflammatory drug dexamethasone can decrease BPD incidence in preterm infants, although not without complications. However, anti-inflammatory treatments that target specific inflammatory mediators have been ineffective to date ${ }^{60}$.

A striking difference between animal models and very preterm infants who develop BPD is that infants are exposed multiple times to factors that alter lung development, such as preterm birth and its associated pathologies, pre-eclampsia, antenatal steroids, infection and/or inflammation, maternal smoking, antenatal and postnatal nutrition, genetic background, sex and ethnicity $^{30}$. Each of these factors changes the response of the preterm lungs to injury. Of note, the infants who are at highest risk of BPD are extremely premature - for example, an infant born at 24 weeks of gestation is born 4 months early ( $65 \%$ of normal gestation length) and therefore has structurally under-developed lungs (FIG. 4) that are often able to support gas exchange but are easily injured. The progression of lung injury to BPD in infants is not the same as the progression that occurs in acute lung injury in adults, as the primary insults (supplemental oxygen and ventilation-mediated injury) in preterm infants can continuously injure the lungs, often for months, depending on the nature of respiratory support and related ventilator strategies (see below). Although these extremely preterm infants can frequently still develop fairly normal lungs, the risk of life-long respiratory and cardiovascular impairment remains high. Insufficient knowledge of the interactions of lung development, injury and repair pathways hampers the development of effective treatments.

Further confounding the epidemiology and pathophysiology of BPD are very preterm infants who initially have almost normal lung function but cannot tolerate modest initial oxygen and ventilation and progress to early respiratory death or severe $\mathrm{BPD}^{39}$. The converse are infants with early severe respiratory failure who respond poorly to surfactant treatment and require very high supplemental oxygen and ventilation pressure, although lung function returns to normal over weeks. These extreme respiratory function patterns indicate that some very premature lungs are particularly sensitive 


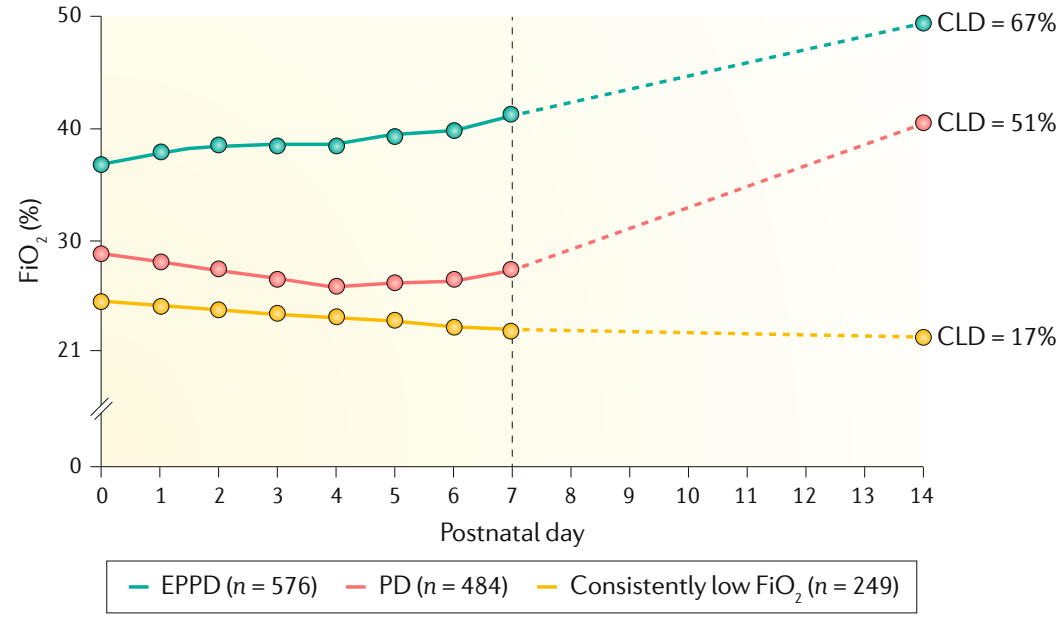

Fig. 2 | Patterns of lung disease in premature infants. Median of the mode fraction of inspired oxygen $\left(\mathrm{FiO}_{2}\right)$ on postnatal days 0 to 7 and on postnatal day 14 , and the frequency of chronic lung disease (CLD) among extremely low gestational age newborns with three patterns of respiratory disease $\left(\right.$ low $\mathrm{FiO}_{2}$, pulmonary deterioration (PD) and early persistent pulmonary deterioration (EPPD)) during the first two postnatal weeks. Delineation of the three patterns of disease is useful for early prognostication of bronchopulmonary dysplasia. Some infants have minimal lung disease and recover quickly, whereas others have EPPD, which requires prolonged, substantial respiratory support from birth. In the third pattern, some infants have initial lung disease that resolves in the first week after birth and is followed by respiratory decompensation (PD) that thereafter requires mechanical ventilation and supplemental oxygen. The dashed lines are the presumed $\mathrm{FiO}_{2}$, as $\mathrm{FiO}_{2}$ was only obtained on postnatal days 7 and 14 . Adapted with permission from REF. ${ }^{48}$, Pediatrics, 123, 1124-1131, Copyright 2009 by the AAP.

to injury and do not heal, whereas others are quite resilient, with resistance to injury and with effective healing. These differences in injury and repair patterns probably result from multiple lung dysmaturity phenotypes that are caused by antenatal exposures, early postnatal exposures and different genetic backgrounds.

\section{Perturbed lung development in BPD}

Chorioamnionitis and inflammation. Inflammation is the common pathway that initiates the lung injury that can progress to BPD. Neonatal respiratory support at delivery can easily injure the premature lung during the transition from fluid-filled lungs to air breathing ${ }^{66}$. Subsequent ventilatory support and oxygen exposure can amplify injury. Some injury may be unavoidable, even with gentle assisted ventilation and surfactant treatment in infants $<30$ weeks gestational age. Subsequent inflammation from sepsis or necrotizing enterocolitis can increase the risk of developing BPD. These postnatal inflammatory exposures are often preceded by two antenatal exposures that are potent effectors of delayed lung development and lung injury. More than $50 \%$ of pregnancies that result in very preterm births ( $<28$ weeks gestational age) have histological evidence of chorioamnionitis ${ }^{67}$ - inflammation of the fetal membranes, maternal decidua and, often, the amniotic fluid - which may be the proximal cause of the preterm labour. Concurrently, in $~ 90 \%$ of pregnancies that are at risk of preterm delivery, maternal antenatal corticosteroid treatment is prescribed to treat RDS and decrease infant mortality ${ }^{68}$. In the complex context of prematurity and BPD, these exposures confound simple interpretations of the causality of BPD.
Chorioamnionitis simply indicates an intrauterine exposure to inflammation, most often associated with Ureaplasma sp., but multiple vaginal commensals and pathogens have also been identified ${ }^{67}$. Chorioamnionitisassociated deliveries may also have 'sterile' inflammation. In clinical practice, the identity of the multiple microorganisms, the duration of fetal exposure and the location of the infection (decidua, amniotic fluid or fetus) are usually unknown. Studies in multiple animal models have demonstrated that fetal breathing exposes the fetal lung to infection and/or inflammation in amniotic fluid and causes lung inflammation and often a fetal inflammatory response ${ }^{69}$. Low-grade lung inflammation induces lung maturation in experimental models. In clinical practice, histologically-confirmed chorioamnionitis is associated with decreased RDS incidence ${ }^{70}$, whereas the presence of Ureaplasma sp. in cord blood is associated with increased incidence of $\mathrm{BPD}$ but no change in the incidence of $\mathrm{RDS}^{71}$. In another clinical series, increased severity of BPD correlated with increased severity of chorioamnionitis ${ }^{72}$. However, a summary meta-analysis of 59 studies could not definitively associate chorioamnionitis with increased BPD risk $^{28}$, perhaps reflecting issues with standardizing the diagnoses of chorioamnionitis and the complex interactions of multiple perinatal risk factors.

Most very preterm fetuses exposed to chorioamnionitis are also exposed to antenatal corticosteroids, which in animal models suppress the inflammation associated with chorioamnionitis, cause an arrest of alveolarization and also induce greater lung maturation than either exposure alone ${ }^{73}$. Tolerance or preconditioning (modulation of the response to a fetal or neonatal exposure by a previous fetal exposure) is a possible reason why single fetal or neonatal exposures in animal models poorly replicate outcomes in preterm infants. Depending on timing and the exposure, a second similar or different exposure can result in a blunted or exaggerated lung injury response. In fetal sheep, an intra-amniotic injection of endotoxin causes fetal lung inflammation. However, prior chronic exposure of pregnant ewes to live Ureaplasma sp. completely blocks the fetal lung response to endotoxins ${ }^{74}$. Similarly, fetal exposure to intra-amniotic lipopolysaccharide (LPS) can protect the newborn from hypotension from intravascular LPS administration ${ }^{75}$. Very preterm infants are exposed to multiple potent antenatal and postnatal inflammatory modulators that modify injury and repair pathways, which is expressed as the clinical variability and severity of BPD.

Alterations of the lung microbiome have been recognized in multiple respiratory disorders. It is conceivable that, similar to chorioamnionitis, alterations in the lung microbiome prime the developing lung to injury ${ }^{76}$. The increasing evidence linking the microbiome in preterm infants to BPD has been analysed in a systematic review $^{77}$, which reported substantial heterogeneity in the six included studies. Microbial dysbiosis may be associated with BPD progression and severity. Most of these infants were born by caesarean section and were exposed to postnatal antibiotics. Novel insights into systems biology based on 'omics' approaches may enable the temporal analysis of the fetal and neonatal lung 
and gut microbiomes in preterm infants, and ultimately permit manipulation to correct dysbiosis and restore a 'healthy' microbial environment.

Vascular abnormalities. Pulmonary hypertension and related pulmonary vascular disease (PVD) have long been recognized as strong contributors to poor survival in preterm infants with $\mathrm{BPD}^{1}$. A diagnosis of pulmonary hypertension that persists beyond the first few months of life has been associated consistently with mortality rates as high as $40-50 \%$, ranging from a report in $1980\left(\mathrm{REF}^{78}\right)$ to a study in $2007\left(\mathrm{REFS}^{79,80}\right)$. Prospective studies have provided echocardiographic evidence of pulmonary hypertension in $14-25 \%$ of preterm infants at 36 weeks post-menstrual age (PMA), with pulmonary hypertension especially prevalent $(29-58 \%)$ in infants with severe $\mathrm{BPD}^{81-83}$.

Alveolus
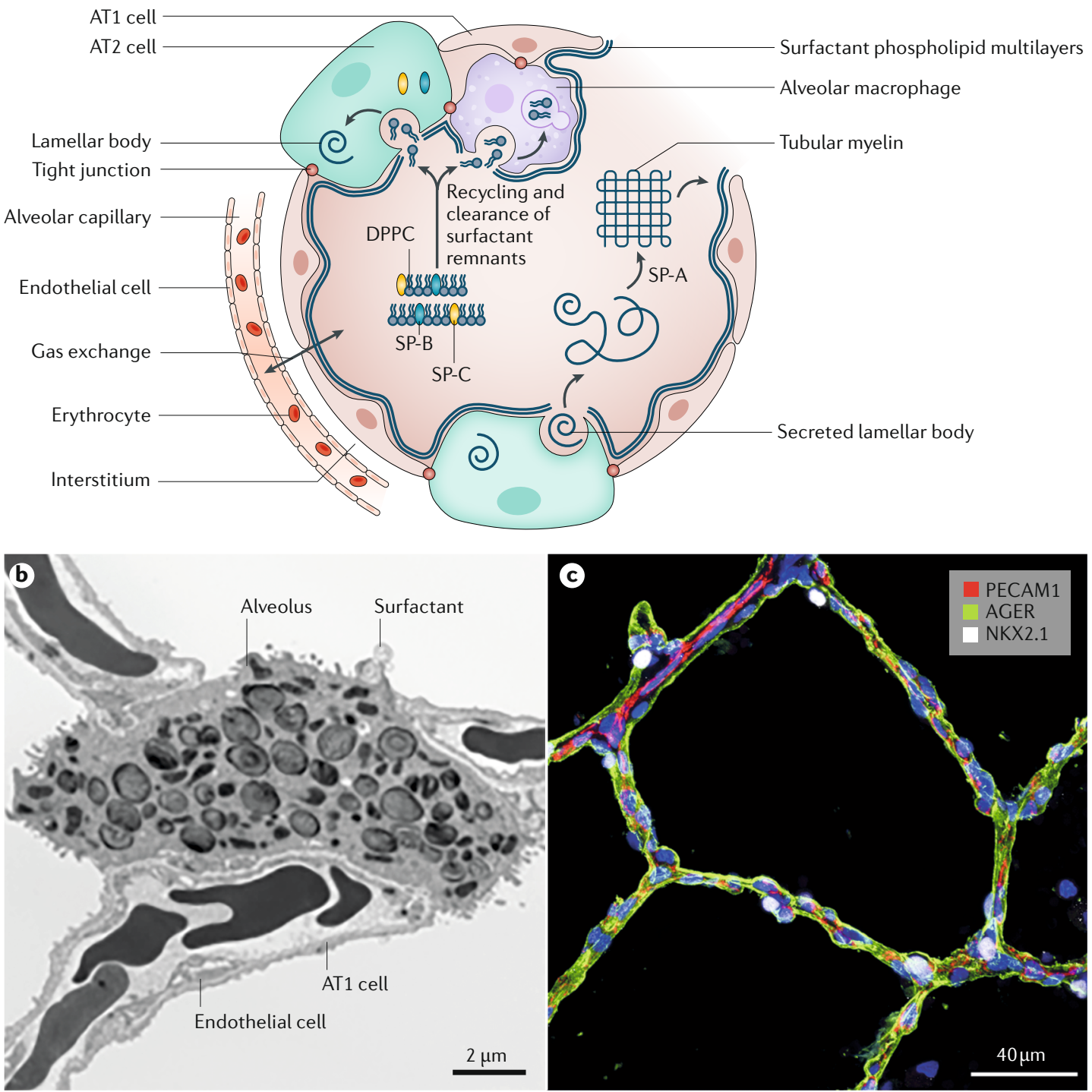

Fig. 3 | Structure of the alveolar gas exchange region. a | Schematic representation of the alveolar unit. Surfactant lipids (such as dipalmitoylphosphatidylcholine (DPPC)) and proteins (such as SP-B and SP-C) are produced by alveolar type 2 (AT2) cells. Surfactant is secreted onto the alveolar surface to reduce surface tension and prevent atelectasis. After secretion, surfactant lipids interact with SP-A to form tubular myelin, from which a multilayered film of surfactant lipids is produced. The surface-active phospholipids reduce surface tension at the air-liquid interface in the alveolus to prevent alveolar collapse. Surfactant lipids and proteins are recycled or degraded, the latter primarily by alveolar macrophages. Intercellular communication between alveolar epithelial cells and macrophages integrates local inflammatory signals, resulting in upregulated expression of cytokines and chemokines in response to pathogens. $\mathbf{b} \mid$ Electron microscopy image revealing the ultrastructure of the alveolus. AT2 cells contain intracellular lamellar bodies that comprise surfactant lipids and proteins. Squamous AT1 cells and endothelial cells form the gas exchange unit. Erythrocytes are seen within the alveolar capillaries of the pulmonary microvasculature. $\mathbf{c}$ |Confocal microscopy image of human alveoli from a 4-year-old child. On immunofluorescence imaging, anti-AGER antibody stains the surface of AT1 cells (green), anti-NKX2.1 antibody stains the nuclei of AT2 cells (white) and anti-PECAM1 antibody stains endothelial cells (red) in the microvasculature. Parts $\mathbf{a}$ and $\mathbf{b}$ adapted from REF. ${ }^{287}$, Springer Nature Limited. 


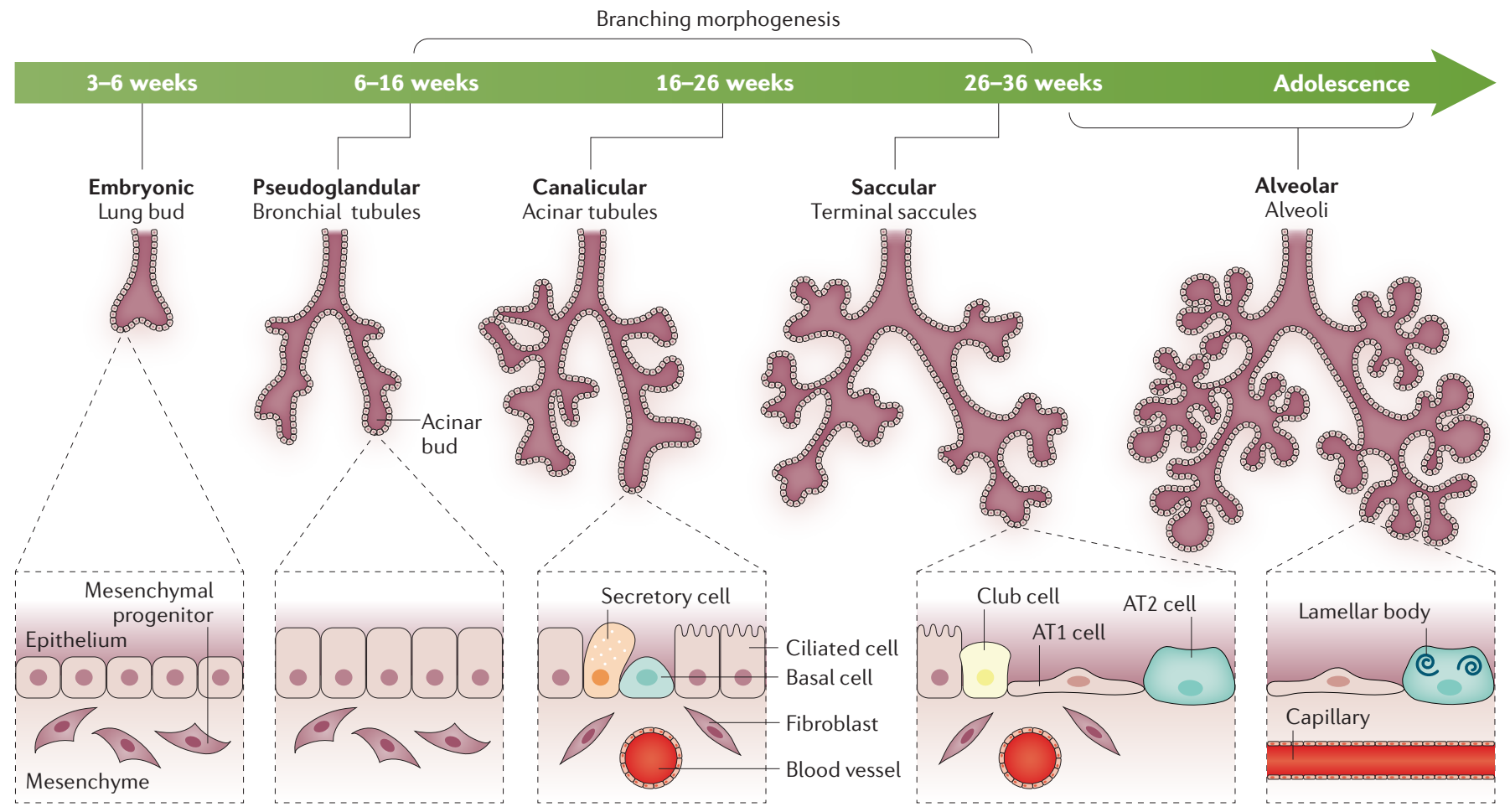

Fig. 4 | Human lung morphogenesis. Schematic of the stages of lung formation from the embryonic stage to alveolarization. Changes in lung structure with advancing gestation are shown. In the embryonic period of lung bud formation, the tracheal primordium forms from the ventral region of the anterior foregut endoderm and separates from the oesophagus. During the pseudoglandular period, the lung buds proliferate and invade the splanchnic mesenchyme in the process of branching morphogenesis to form the airways and peripheral acinar buds, the latter forming the alveoli later in development. During the saccular stage, epithelial cells lining conducting airways differentiate, producing basal, goblet, ciliated and other secretory cells, which are distinct from the epithelial cells lining the peripheral saccules, namely the cuboidal pre-alveolar type 2 (AT2) cells and squamous AT1 cells. In the saccular-alveolar transition, the peripheral saccules further dilate and the surface is increasingly covered by AT1 cells as the gas exchange region expands. AT2 cells differentiate and produce increasing amounts of surfactant lipid and proteins, which are stored in lamellar bodies. Lung growth continues until adolescence. Adapted with permission from REF. ${ }^{288}$, Oxford University Press.

Thus, despite major advances in perinatal and neonatal intensive care unit (NICU) care with improved survival and changes in the nature of BPD over the decades, pulmonary hypertension continues to be a prominent clinical challenge in the pathobiology and outcomes of infants with BPD.

There has been growing awareness in the past decade that pulmonary hypertension in premature infants encompasses several PVD phenotypes (BOX 2), including the fairly high incidence of sustained elevation of pulmonary vascular resistance (PVR) after birth that can cause profound hypoxaemia due to extrapulmonary right-to-left shunting across the foramen ovale and/or ductus arteriosus (persistent pulmonary hypertension of the newborn (PPHN)) with a slow adaptation or 'delayed vascular transition ${ }^{35,84-86}$. Although more often considered in near-term or term infants with hypoxaemia at birth, PPHN also occurs in preterm infants and is negatively associated with gestational age (FIG. 6a). Severe parenchymal lung disease can contribute to elevated PVR; however, abnormalities of the pulmonary vasculature can also contribute to high PVR, especially in the setting of antenatal stresses, such as oligohydramnios and prolonged premature rupture of membranes ${ }^{84-87}$.
Importantly, echocardiography-confirmed delayed pulmonary vascular transition ${ }^{86}$ and early pulmonary hypertension $^{88}$ are associated with a higher risk of the subsequent development of BPD, late pulmonary hypertension and higher mortality ${ }^{86,88}$. Experimental studies support epidemiological findings that adverse intrauterine stimuli are sufficient to impair vascular growth and induce longstanding and severe pulmonary hypertension independent of postnatal factors, such as hyperoxia and ventilator-induced injury ${ }^{89}$.

In addition to the important effect of pulmonary hypertension, preclinical and clinical studies suggest that early disruption of lung vascular growth and function can impair growth of the distal airspace (the 'vascular hypothesis' of BPD ${ }^{90-93}$. Furthermore, strong clinical studies have shown that abnormal placental vascular structure and placental hypoperfusion on histopathology are strikingly associated with neonatal outcomes of IUGR and susceptibility to subsequent development of BPD and pulmonary hypertension ${ }^{94,95}$. Prospective studies have shown that antenatal factors assessed on the first day of life ${ }^{30,96}$, cord blood biomarkers of impaired angiogenesis ${ }^{97-99}$ and early echocardiography findings of pulmonary hypertension shortly after birth are strongly 

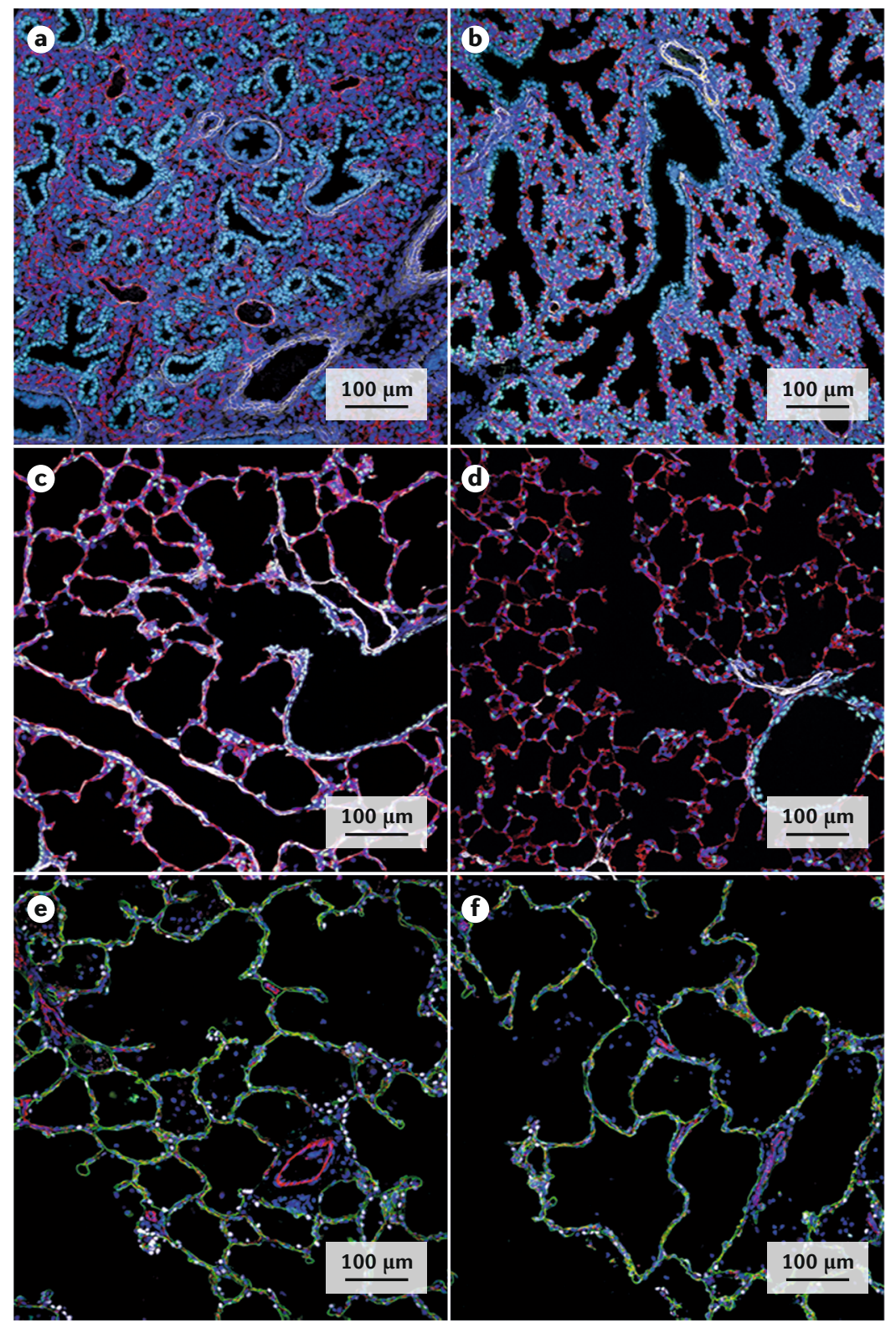

Fig. 5 | Structural changes in the lung during development and in BPD. a-d | Developmental changes in lung structure revealed by immunofluorescence and confocal microscopy of a mouse lung at the pseudoglandular (embryonic day 16.5 (E16.5); part a), canalicular (E18.5; part b), saccular (postnatal day 3 (P3); part c) and alveolar (P28; part d) stages of lung development. The transcription factor NKX2.1 (light blue) is required for lung morphogenesis and is expressed by the epithelial cells lining the trachea, bronchi and peripheral lung tubules. An antiEMCN antibody stains endothelial cells (red) in the developing microvasculature. An anti-ACTA2 antibody stains smooth muscle cells (white) in pulmonary vessels in the bronchial, submucosal and alveolar regions. Smooth muscle actin (ACTA2) staining is prominent on $\mathrm{P} 3$ during active alveolar formation (part $\mathbf{c}$ ) and is less prominent on P28 (part d). e-f | Simplification of alveolar structure in bronchopulmonary dysplasia (BPD). Confocal microscopy images of a section of the lung of a healthy infant (part $\mathbf{e}$ ) and that of an infant with BPD (part $\mathbf{f}$ ) at $\sim 3$ years of age. Alveolar surfaces are primarily lined by squamous alveolar type 1 (AT1) cells, which are stained with anti-AGER antibodies (green). An anti-PECAM1 antibody stains endothelial cells (red) in pulmonary capillaries. An anti-NKX2.1 antibody selectively stains AT2 cells (white). Alveoli form by septation, creating numerous small saccules in the normal lung (part e), whereas the alveoli are enlarged or 'simplified' in the lungs of an infant with BPD (part f), causing loss of the alveolar-capillary gas exchange surface area. linked to the risk of BPD, pulmonary hypertension, prolonged NICU hospitalization and late respiratory outcomes in childhood ${ }^{83,100}$ (FIG. 6b-d). Thus, antenatal determinants not only cause abnormalities of short-term respiratory function, as reflected by NICU course, but early injury in utero causes sustained disruption of lung and pulmonary vascular structure throughout infancy, reflecting the adverse effect of fetal programming ${ }^{89,101}$ (FIC. 1). Antenatal stresses, including chorioamnionitis and pre-eclampsia (with or without IUGR), in preterm neonates contribute to BPD risk ${ }^{102-104}$, although data from animal models of mechanisms linking antenatal stress to BPD pathogenesis and late cardiorespiratory outcomes are limited.

\section{Diagnosis, screening and prevention Diagnosis}

BPD was first described in moderately preterm infants in an era when supplemental oxygen was the primary therapy for severe $\mathrm{RDS}^{1}$ and mortality was $>50 \%$. Since then, the definition of BPD has evolved (TABLE 1), in large part because of the improved survival of extremely preterm infants and the new modalities used to support them (BOX 1).

Current challenges in defining BPD. For most diseases, diagnosis is an end in itself. For preterm infants, a diagnosis of BPD is more akin to a functional lung assessment at the time a preterm infant is expected to transition out of hospital (similar to how forced expiratory volume in 1 second (FEV1) is used in conditions such as cystic fibrosis ${ }^{105}$. More than 30 years after BPD was defined by Shennan et al ${ }^{106}$, how well a BPD diagnosis can predict pulmonary outcomes in infancy and childhood, and how those long-term pulmonary outcomes should be defined, remain primary research and clinical questions ${ }^{107}$.

The populations at risk and respiratory treatment strategies have also evolved since publication of the 2001 National Heart, Lung, and Blood Institute (NHLBI) workshop report ${ }^{108}$. The number and survival of the smallest and most preterm infants has increased, and some NICUs now routinely care for babies born at 22 weeks of gestation ${ }^{24,26}$. Respiratory care practices have also changed, as recently documented by the Prematurity and Respiratory Outcomes Program (PROP) cohort of 765 surviving infants of $<29$ weeks of gestation ${ }^{109}$. In the PROP cohort, 359 infants (47\%) were treated with nasal cannula flow at 36 weeks PMA, including 95 infants $(12 \%)$ on flow with room air. This creates new problems for BPD classification, as it is not possible to differentiate whether flow is needed for control of breathing or for lung parenchymal injury. Data capture outside a rigorous research environment is also challenging. In the database maintained by the National Perinatal Registry of the Netherlands, only $67 \%$ of preterm infants were correctly categorized according to the NHLBI workshop definition ${ }^{110}$, owing predominantly to a false-negative diagnosis rate of $31 \%$, including $9 \%$ with severe BPD. A bedside analysis of the 'shift' of the oxyhaemoglobin dissociation curve (accomplished through simultaneous measurement of $\mathrm{FiO}_{2}$ and peripheral saturation at a 
Box 2 | Pulmonary vascular disease phenotypes of prematurity

\section{Early (first 2 weeks after birth)}

- Persistent pulmonary hypertension of the newborn syndrome: hypoxaemic respiratory failure with extrapulmonary right-to-left shunt

- Delayed vascular transition

- Mild pulmonary hypertension as a biomarker for risk of bronchopulmonary dysplasia or late pulmonary hypertension

\section{Late (weeks to months after birth)}

- Sustained need for high levels of respiratory support (fraction of inspired oxygen, ventilation), often with recurrent exacerbations or cyanotic episodes

- Pulmonary hypertension after discharge from neonatal intensive care unit, with persistent respiratory problems (such as intermittent hypoxia and obstructive sleep apnoea), acute viral infections and other symptoms

Chronic (months to years after birth)

- Persistent echocardiographic abnormalities during infancy, childhood and adolescence

- Exercise intolerance during childhood and adolescence

- High normal ('borderline') pulmonary hypertension and abnormal cardiac size and structure in young adults to account for other environmental confounders, such as smoking and viral infections.

The 2001 NHLBI workshop definition took the important step of including a BPD severity scale ${ }^{108}$, and infants with severe BPD were subsequently found to have higher mortality and rates of adverse outcomes after discharge than those with mild or moderate disease $^{114}$. However, the definition of severe BPD was overly broad, and combined infants receiving $31 \%$ oxygen by low flow nasal cannula with infants on high ventilator settings and receiving drugs for pulmonary hypertension. This issue was partly addressed by the NHLBI 2018 revision, which reserved grade $3 \mathrm{BPD}$ for infants receiving positive pressure or nasal cannula flow $>31 / \mathrm{min}$ in addition to oxygen ${ }^{3}$. To better refine the approach to defining disease severity for clinical care and research, the BPD Collaboration Group recommended that severe BPD be further subclassified, with infants receiving only nasal cannula oxygen at 36 weeks PMA defined as type 1 severe BPD and infants requiring mechanical ventilation defined as type 2 severe $\mathrm{BPD}^{115}$. The Canadian Neonatal Network added weight to this idea by showing that a more severe BPD diagnosis (that is, at 36 weeks PMA requiring oxygen plus respiratory support versus oxygen only) better predicted serious respiratory morbidity at 18-22 months ${ }^{14}$. However, because of the low incidence of type 2 severe BPD in any single centre, long-term outcomes remain poorly understood for this population. In addition, although pulmonary hypertension is a common comorbidity and probably contributes to the pathophysiology of severe BPD, it has not been incorporated in any existing definition ${ }^{116}$.

The most severe BPD leads to early mortality prior to 36 weeks PMA. Although this outcome was estimated to occur in $3 \%$ of the PROP cohort ${ }^{117}$, these especially high-risk infants have been inconsistently classified using existing definitions. The 2018 NICHD workshop definition proposed a grade 3A BPD for infants with early death between 14 days postnatal age and 36 weeks PMA from persistent parenchymal lung disease and respiratory failure that is not attributable to other neonatal morbidities. Further work and validation of the NICHD BPD risk calculator ${ }^{49}$ is needed to better identify this population of infants as early as possible in their clinical course (ideally before 14 days), as a particularly high-risk group for novel therapies.

Finally, future BPD definitions should provide strong specificity in addition to sensitivity. In the PROP cohort, about $50 \%$ of infants with persistent respiratory disease at 1 year of life had no or mild BPD at 36 weeks PMA ${ }^{96}$. Improving predictive value will require better objective measurements and biomarkers of lung injury and BPD and the ability to incorporate antenatal factors, such as IUGR, maternal hypertension, maternal smoking and male sex.

\section{Screening and prevention}

Biomarkers. Prevention of BPD and long-term cardiorespiratory impairment remains elusive, making identification of early predictive BPD biomarkers to guide new therapies a laudable goal. Preterm infants with differing risks of developing BPD have been enrolled in early 


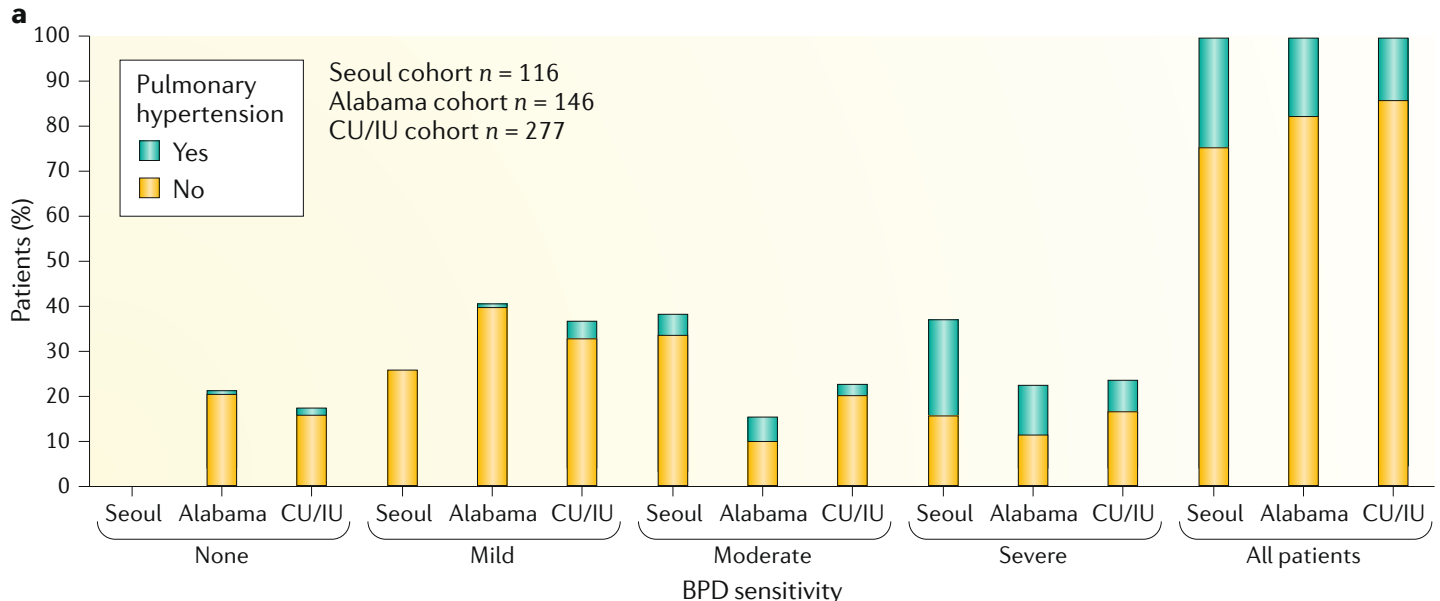

b

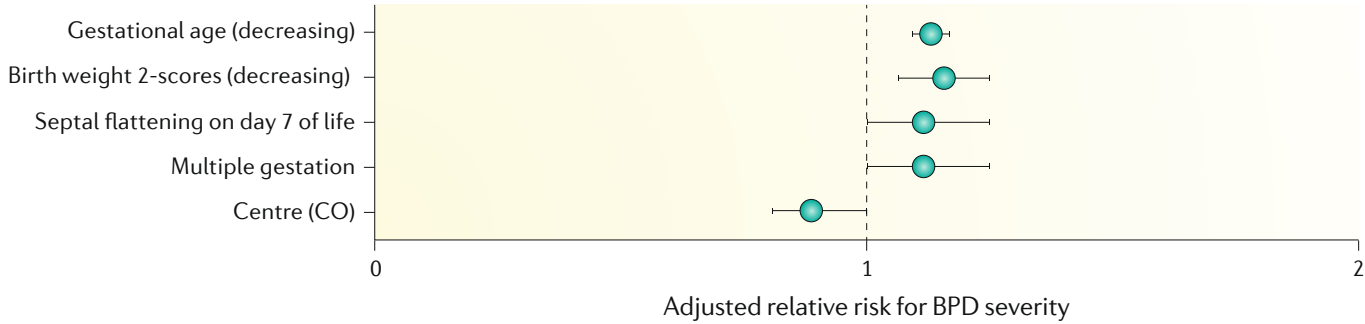

Septal flattening on day 7 of life

Right ventricular dilation on day 7 of life

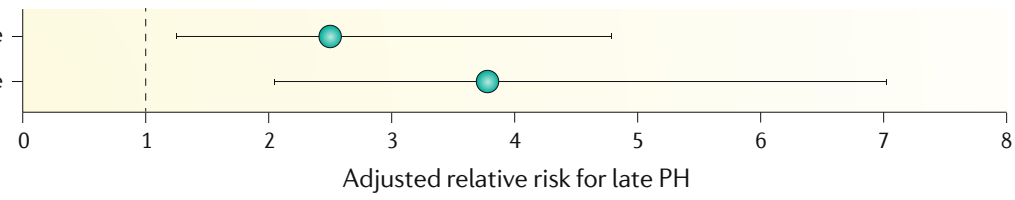

d

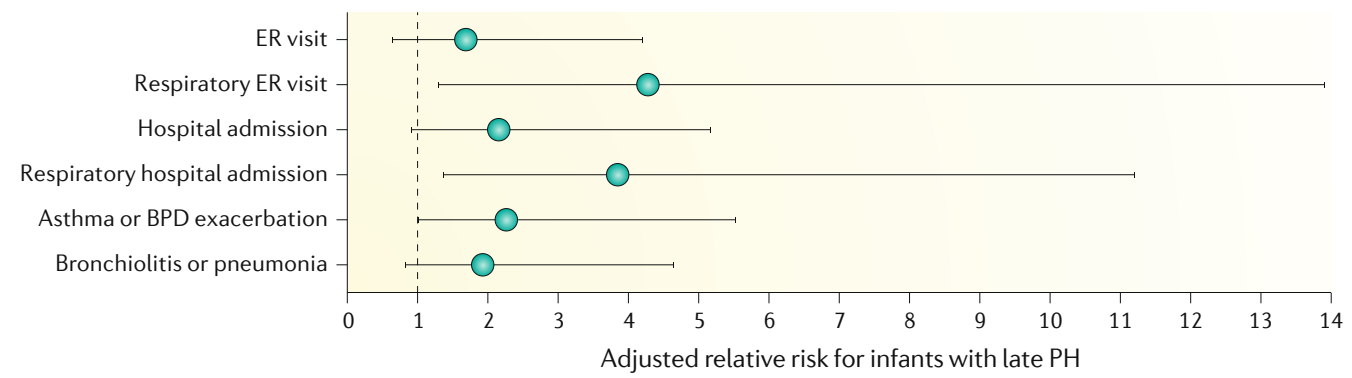

Fig. 6 | Pulmonary vascular disease in BPD. a | Prevalence of pulmonary hypertension in preterm infants, as assessed by echocardiography at 36 weeks post-menstrual age. Pulmonary hypertension was diagnosed in $14-25 \%$ of preterm infants in different cohorts (Seoul, Korea ${ }^{81}$; Alabama, USA ${ }^{82}$; and Colorado (CO) and Indiana (CU/IU), USA ${ }^{83}$ ), with the highest incidence of pulmonary hypertension associated with severe bronchopulmonary dysplasia (BPD). b-d | Early pulmonary vascular disease, as assessed by echocardiography on postnatal day 7 , is strongly associated with adverse outcomes, including severe BPD (part b), risk of pulmonary hypertension $(\mathrm{PH})$ at 36 weeks (part $\mathbf{c}$ ) and risk of respiratory disease during the first 2 years of life ${ }^{80,83,100}$ (part d). ER, emergency room. Part a adapted with permission from REF ${ }^{80}$, Elsevier. Parts $\mathbf{b}$ and $\mathbf{c}$ adapted with permission from REF. ${ }^{83}$, of the American Thoracic Society. Copyright 2019 American Thoracic Society. Part d adapted with permission from REF. ${ }^{100}$, of the American Thoracic Society. Copyright 2019 American Thoracic Society.

prevention trials, sometimes with adverse consequences. A prime example is the trials of high-dose dexamethasone, which was later found to be associated with a higher risk of cerebral palsy and intestinal perforation ${ }^{118}$. BPD is a heterogeneous disease resulting from multiple pathophysiological processes, with varying magnitudes of impairment of alveolar septation, lung fibrosis and abnormal vascular development and remodelling ${ }^{119}$. Failure to identify subpopulations at highest risk and with distinct mechanisms of disease (endotypes) contributes to exposure to therapies that are unlikely to benefit or may cause harm to individual patients, as well as scepticism about biologically plausible therapies that may benefit a subset of at-risk infants ${ }^{120,121}$. Identifying infants at high risk of BPD at birth is possible, as was shown for the PROP cohort ${ }^{96}$. Infants who have a persistent need for respiratory support beyond 10-14 days of age are at the highest risk of a BPD diagnosis at 36 weeks $^{96,122}$. Although persistent oxygen requirement in the first weeks of life may be a clinical predictor of 
later BPD, it provides little information about disease endotypes or therapeutic interventions that may benefit individual patients.

A good BPD biomarker should be easily measurable, be affordable, use a reliable assay with high analytical sensitivity and specificity, predict later lung disease or serve as a surrogate for long-term respiratory outcomes, elucidate underlying pathophysiological mechanisms (thereby refining BPD risk by endotypes) and be validated in diverse replication cohorts. A validated BPD biomarker offers the potential to reduce health-care costs and the risks of broadly applying ineffective therapies. Biomarkers may be valuable for earlier diagnosis of BPD, enabling initiation of therapies when they may be more effective or avoidance of therapies and their potential hazards ${ }^{119}$. A validated biomarker may also facilitate the development of tailored, personalized therapeutic approaches and improve patient endotyping prior to study randomization, thereby enhancing safety and efficacy of randomized controlled trials of novel therapies. Conversely, biomarker testing may increase screening costs and result in fewer infants who are eligible for trials, thus requiring larger multicentre collaborative efforts ${ }^{120}$.

Despite their promise, there are many challenges to identifying BPD biomarkers. An important consideration is the optimal biomarker source or specimen type, which could include DNA, microRNA, amniotic fluid, tracheal aspirate, blood, urine and stool. For example, the levels of IL- $1 \beta$, IL-6, CXCL10 and uric acid are substantially higher in tracheal aspirate but not in plasma of patients with moderate and severe BPD than in that of infants with no BPD ${ }^{123}$. The best approach to normalizing a sample for volume collected or dilution factors in some specimen types, such as tracheal aspirate and urine, must be determined. The optimal timing for measurement of the biomarker and therefore therapeutic intervention (at birth and/or the first hours, days or weeks of life) must also be ascertained. Normal patterns of biomarker expression must be determined across a range of gestational and postnatal ages to identify patterns that predict disease or deviation from normal development. Determining the appropriate control group is a unique challenge for research in the preterm population. Healthy term infants may not represent an appropriate control when biomarker expression changes with developmental maturation. It is not always clear whether the absolute value or a change in biomarker level over time will yield the most predictive signal or whether to apply cut-off values or quartiles for risk stratification. Last, interactions among various biomarkers must be taken into account; for example, the balance of pro-inflammatory and anti-inflammatory cytokines or pro-oxidants and antioxidants may be a better predictor of BPD and later respiratory morbidity than a single biomarker of inflammation or oxidative stress.

Various biomarkers have been studied in association with BPD but have yet to be validated as predictors of important long-term outcomes (see TABLE 2 for a nonexhaustive list of BPD biomarkers). Ideal biomarkers for BPD will target causal pathways that have been implicated in BPD pathogenesis ${ }^{120,121}$, such as biomarkers of

\section{Table 1 | Definitions of BPD}

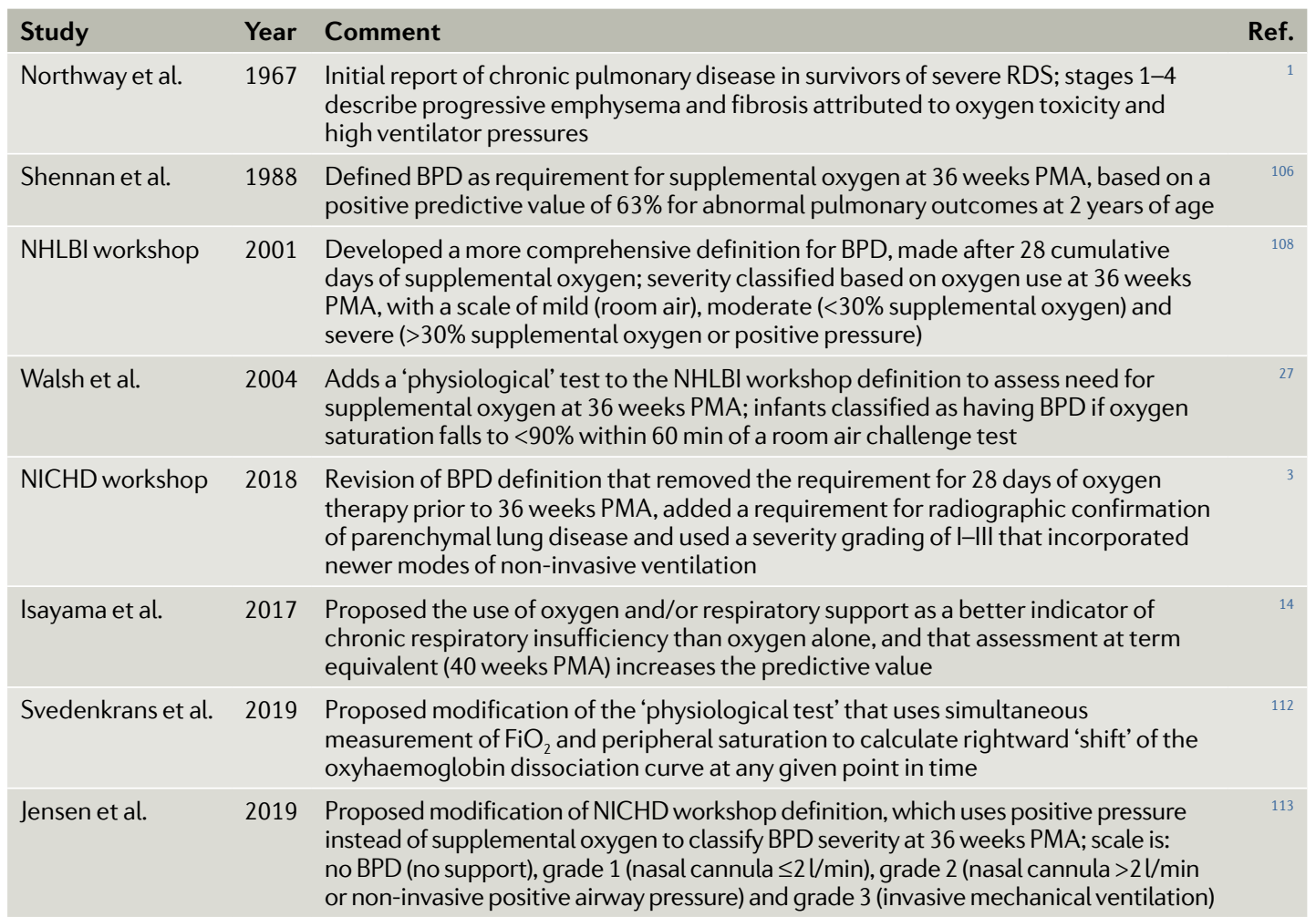

BPD, bronchopulmonary dysplasia; $\mathrm{FiO}_{2}$, fraction of inspired oxygen; NHLBI, National Heart, Lung, and Blood Institute; NICHD, Eunice Kennedy Shriver National Institute of Child Health and Human Development; PMA, post-menstrual age; RDS, respiratory distress syndrome. 
endothelial injury and dysfunction ${ }^{124,125}$; growth factors and biomarkers of altered angiogenesis ${ }^{6,126-131}$; biomarkers of epithelial injury and fibrosis ${ }^{132-135}$; biomarkers of nitric oxide deficiency or insufficiency ${ }^{120,136-140}$; biomarkers of oxidative stress, oxidant and antioxidant capacity, and redox status ${ }^{141-143}$; oxyradical biomarkers $^{141-144}$; biomarkers of inflammation and host immune responses ${ }^{129,145,146}$; genomic, epigenomic, metabolo$\mathrm{mic}^{147}$, microbiomic ${ }^{148}$, methylomic and transcriptomic biomarkers ${ }^{119,149}$; lung function measures ${ }^{119,150}$; MRI of the lungs for measurement of perfusion, blood flow, ventilation, gas exchange, mechanics and early changes in alveolar structure ${ }^{119}$; and biochemical ${ }^{151}$ and physiological biomarkers of vascular injury and pulmonary hypertension $^{80,82,123,140,152}$. A 2013 systematic review identified serum KL6, CC16 and neutrophil gelatinaseassociated lipocalin, and end-tidal carbon monoxide (etCO), as performing well in predicting BPD in preterm infants ${ }^{153}$. Identification of early markers of endothelial or vascular injury may lead to mechanistic interventions to support normal vascular and alveolar growth and prevent BPD.

Rare single-gene diseases with a BPD-like phenotype probably occur in preterm infants. In the absence of a high suspicion of such a single-gene pulmonary disease, genomic analysis is unlikely to be useful as a screening biomarker. To date, only a few underpowered genomewide association studies (GWAS) on BPD have been published $^{149,154}$ (reviewed in REF. ${ }^{37}$ ). Very large genomic studies with international or multicentre participation and including multiple ethnicities, antenatal risk factors and careful definition of phenotypes, may provide important clues about pathogenetic pathways and
BPD endotypes. Although identification of biomarkerdefined BPD endotypes holds promise for a new era of BPD prevention research, none of the biomarkers discussed above have been effectively used. Harnessing the promise of biomarkers will depend on identification and validation of affordable, reproducible, point-of-care or rapid biomarker tests and advances in computational biology and artificial intelligence ${ }^{155}$.

\section{Management \\ Short-term management}

In the absence of effective interventions to prevent preterm birth, treatment strategies have focused on reducing exposure to ventilation using an endotracheal tube, to avoid or minimize long-term lung and brain damage in preterm infants (TABLE 3). Of note, some clinical studies have suggested that BPD is an independent risk factor for poor neurodevelopmental outcomes, even in the absence of definite brain injuries, such as intraventricular haemorrhage or hypoxic ischaemic encephalopathy ${ }^{156,157}$. For example, motor functions, cognitive development and academic progress are worse in preterm infants with BPD than in those without BPD ${ }^{158}$, possibly because strategies to protect the lungs might also be neuroprotective.

Avoiding intubation and ventilation. Attempts to avoid lung injury in extremely preterm infants have led to recommendations to avoid intubation and ventilation in the first minutes of life and a preference for non-invasive respiratory support ${ }^{159}$. Besides nasal continuous positive airway pressure (NCPAP), nasal intermittent positive pressure ventilation (NIPPV) and

Table 2 | Selected biomarkers associated with BPD pathogenesis

\begin{tabular}{|c|c|c|}
\hline Category or causal pathway & Biomarker $^{a}$ & Refs \\
\hline $\begin{array}{l}\text { Endothelial injury and } \\
\text { dysfunction }\end{array}$ & Circulating endothelial progenitor cells & 124 \\
\hline Growth and angiogenesis factors & $\begin{array}{l}\text { VEGF and VEGFR, endoglin (CD105), angiopoietin 1, endostatin, } \\
\text { PIGF and EMAP II }\end{array}$ & $125-128,130,131$ \\
\hline Epithelial injury and fibrosis & KL6, MMP9, TIMP1, TGF $\beta 1$ and NGAL & $82,132-135$ \\
\hline $\begin{array}{l}\text { Nitric oxide deficiency and } \\
\text { insufficiency }\end{array}$ & $\mathrm{NO}_{x}$ and citrulline & $120,136-140$ \\
\hline Oxidative stress, redox status & $\begin{array}{l}\text { Elastase, myeloperoxidase, xanthine oxidase, catalase, total } \\
\text { sulfhydryls, carbonyls, 3-chlorotyrosine, malondialdehyde and } \\
\text { 3-nitrotyrosine }\end{array}$ & $144,284-286$ \\
\hline $\begin{array}{l}\text { Inflammation and host immune } \\
\text { responses }\end{array}$ & Inflammatory biomarker panels and hyaluronan & $129,145,146$ \\
\hline Omics & $\begin{array}{l}\text { Genomics, epigenomics, metabolomics, microbiomics, methylomics } \\
\text { and transcriptomics }\end{array}$ & $34,119,147-149,243$ \\
\hline $\begin{array}{l}\text { Vascular injury and pulmonary } \\
\text { hypertension }\end{array}$ & BNP and NT-proBNP and echocardiographic measurement & $83,123,140,151$ \\
\hline Lung function & $\begin{array}{l}\text { Dynamic compliance, airway resistance, functional residual } \\
\text { capacity, etCO and exhaled NO }\end{array}$ & $82,119,150$ \\
\hline Lung structure & Imaging by hyperpolarized gas MRI and helium-3 MRI & 119 \\
\hline \multicolumn{3}{|c|}{$\begin{array}{l}\text { BNP, B-type natriuretic peptide; BPD, bronchopulmonary dysplasia; EMAP II, endothelial monocyte-activating polypeptide II; } \\
\text { etCO, end tidal carbon monoxide; KL6, Kerbs von Lungren } 6 \text { antigen; MMP9, matrix metalloproteinase } 9 \text {; NGAL, neutrophil } \\
\text { gelatinase-associated lipocalin; NO, nitric oxide; NO }{ }_{x} \text {, nitric oxide metabolites; NT-proBNP, N-terminal pro-B-type natriuretic } \\
\text { peptide; PIGF, placental growth factor; TGF } \beta 1 \text {, transforming growth factor } \beta 1 \text {; TIMP1, tissue inhibitor of metalloproteinase } 1 \text {; } \\
\text { VEGF, vascular endothelial growth factor; VEGFR, VEGF receptor. }{ }^{2} \text { Selected biomarkers that have been studied in human infants } \\
\text { with and without BPD. }\end{array}$} \\
\hline
\end{tabular}


Table 3 | Interventions to minimize lung injury in premature infants

\begin{tabular}{|c|c|c|c|c|}
\hline Intervention & Rationale & Level of evidence & Clinical implications & Refs \\
\hline Caffeine & $\begin{array}{l}\text { Reduced time on ventilator } \\
\text { leading to less lung damage }\end{array}$ & $\begin{array}{l}\text { High quality: large RCT with } \\
\text { long-term follow-up }\end{array}$ & $\begin{array}{l}\text { Recommended for use in extremely } \\
\text { preterm infants at the doses used in the } \\
\text { CAP trial }\end{array}$ & 174,175 \\
\hline Vitamin A & $\begin{array}{l}\text { Low levels seen in preterm } \\
\text { infants; vitamin A required } \\
\text { for normal lung growth }\end{array}$ & $\begin{array}{l}\text { Moderate quality: meta-analysis } \\
\text { suggests a small reduction in rates } \\
\text { of death or BPD }\end{array}$ & $\begin{array}{l}\text { Depends on local incidence of } \\
\text { BPD; trade-off between the modest } \\
\text { reduction in BPD and acceptability of } \\
\text { intramuscular treatment }\end{array}$ & 261 \\
\hline $\begin{array}{l}\text { Targeting lower oxygen } \\
\text { saturation levels } \\
\text { throughout NICU stay }\end{array}$ & $\begin{array}{l}\text { Avoidance of high oxygen } \\
\text { levels may reduce BPD }\end{array}$ & $\begin{array}{l}\text { High quality: individual patient } \\
\text { data meta-analysis of five high- } \\
\text { quality RCTs }\end{array}$ & $\begin{array}{l}\text { Targeting higher oxygen saturations } \\
(91-95 \%) \text { improves survival rates } \\
\text { without increasing risk of BPD }\end{array}$ & 198 \\
\hline Hydrocortisone & $\begin{array}{l}\text { Avoids adverse } \\
\text { neurodevelopmental effects } \\
\text { of dexamethasone }\end{array}$ & $\begin{array}{l}\text { Moderate quality: small RCTs, } \\
\text { imprecise estimates of safety and } \\
\text { efficacy }\end{array}$ & $\begin{array}{l}\text { Promising, but more research into } \\
\text { neurodevelopmental outcomes is } \\
\text { required }\end{array}$ & $182-188$ \\
\hline With surfactant & $\begin{array}{l}\text { Better distribution of steroids } \\
\text { and reduced systemic effects }\end{array}$ & $\begin{array}{l}\text { Low quality: small RCTs, imprecise } \\
\text { estimates of safety and efficacy }\end{array}$ & $\begin{array}{l}\text { Very promising, but more research } \\
\text { required }\end{array}$ & 191 \\
\hline
\end{tabular}

BPD, bronchopulmonary dysplasia; CAP, Caffeine for Apnea of Prematurity; RCT, randomized controlled trial.

high-flow nasal cannulae have become more popular, but the efficacy of these respiratory support methods in reducing the rates of BPD has been modest ${ }^{160-163}$. Exogenous surfactant therapy improves neonatal outcomes, including reducing mortality, air leak and the combined outcome of death or $\mathrm{BPD}^{164,165}$. Traditionally, surfactant has been administered using an endotracheal tube. A number of techniques have been developed for the delivery of surfactant to infants who are managed with NCPAP, including less-invasive surfactant administration (LISA) ${ }^{166}$, which uses McGill's forceps to guide a feeding tube between the vocal cords. Alternatively, minimally invasive surfactant therapy (MIST) ${ }^{167}$ uses a firmer vascular catheter and does not require forceps. A meta-analysis of six randomized trials in 895 infants showed that surfactant delivered using a thin catheter reduced the composite outcome of death or BPD compared with conventional intubation for surfactant delivery $^{168}$. Some NICUs have responded to this evidence enthusiastically and implemented routine administration of surfactant to infants on NCPAP. Others have been more cautious and await the results of ongoing larger randomized controlled trials to determine the place of this therapy in modern neonatal intensive care $^{169}$. Alternative approaches to surfactant administration, such as nebulization ${ }^{170}$ and administration into the pharynx or via a laryngeal mask airway ${ }^{171}$, have potential but require further rigorous assessment before general use.

Based on evidence that extremely preterm infants exhibit persistent surfactant dysfunction, late or repeated surfactant administration has been explored. Although the TOLSURF trial showed no difference in survival at
36 weeks between infants without $\mathrm{BPD}$ receiving late surfactant administration versus no surfactant, there was a trend toward improvement at 40 weeks (with >20\% decrease in the number infants requiring respiratory support at 40 weeks) and a substantial decrease in the number of infants who required home respiratory support during the first year ${ }^{172,173}$, an issue that is very important for parents because of the level of stress they experience.

Caffeine. Treatment with respiratory stimulants, such as caffeine, is the best-evaluated treatment for reducing BPD risk. The Caffeine for Apnea of Prematurity (CAP) trial demonstrated that caffeine administration reduces the risk of BPD and shortens the duration of ventilation with an endotracheal tube and exposure to supplemental oxygen ${ }^{174}$. Importantly, these short-term benefits are followed by an improvement in cognitive and motor outcomes in infants at 18 months of age ${ }^{175}$. Important questions about caffeine remain, including whether a higher dose and prolonged treatment lead to further improvements in important outcomes.

Corticosteroids. Antenatal steroid therapy represents a major advance in perinatal care, which has resulted in reduced mortality, respiratory distress syndrome, intraventricular haemorrhage and necrotizing enterocolitis, although it does not reduce rates of $\mathrm{BPD}^{176}$.

Corticosteroids are powerful downregulators of inflammation and have been widely used postnatally to prevent and treat BPD. Concerns about adverse effects of these agents, such as neurodevelopmental impairment, mostly with dexamethasone, in the early 2000 s led to recommendations against their use ${ }^{177}$. Further evidence 
has emerged suggesting that the benefits may outweigh the dangers when the risk of BPD is high $(>50 \%)^{178}$. In a small randomized controlled trial, a short course of dexamethasone delivering a lower initial and total dosage than used previously led to a reduction in the duration of ventilation and was not associated with longterm neurodevelopmental impairment ${ }^{179,180}$. The current decade has seen the pendulum swing back in favour of the prescription of corticosteroids. A European survey showed that $13.9 \%$ of infants born between 24 and 29 weeks of gestation were prescribed postnatal corticosteroids $^{181}$. However, substantial variation in prescription occurred in practice (3.1-49.4\%) across the regions surveyed, which was not explained by neonatal characteristics. The authors suggest that this may reflect different interpretations of the evidence, in particular for infants at highest risk of BPD.

Other attempts to improve the risk/benefit ratio of steroid therapy have involved evaluation of other corticosteroids and routes of administration. Based on extensive studies by Watterberg et al. ${ }^{104,182-184}$, the PREMILOC study compared the early use of 10 days of hydrocortisone therapy to placebo in 523 infants $<28$ weeks of gestation ${ }^{185}$. Unfortunately, the trial was stopped at $66 \%$ of planned recruitment because of financial considerations. The primary outcome, survival without $\mathrm{BPD}$, was higher with hydrocortisone treatment than with placebo $(60 \%$ versus $51 \%, P=0.04)$. Follow-up of survivors at 2 years of age revealed no important differences in rates of neurodevelopmental impairment ${ }^{186}$. A smaller trial evaluated the effect of hydrocortisone administered for 22 days in 372 infants born $<30$ weeks of gestation who were ventilator-dependent between day 7 and day 14 of life ${ }^{187}$. There were no important differences in rates of death or BPD between the hydrocortisone and placebo groups. The most recent individual patient data (IPD) meta-analysis including four randomized controlled trials (982 infants) suggests that early low-dose hydrocortisone therapy increases survival without BPD in very preterm infants, despite an increased risk of intestinal perforation (when given in association with indomethacin) and late-onset sepsis ${ }^{188}$.

Avoidance of systemic administration underpinned the NEuroSIS trial of inhaled budesonide ${ }^{189,190}$. Although

\section{Box 3 | Lung pathophysiology of bronchopulmonary dysplasia}

\section{Central airways \\ - Tracheomalacia \\ - Subglottic stenosis, cyst \\ - Granulomas \\ - Bronchomalacia \\ - Bronchial stenosis}

Small airways

- Structural remodelling

Mucus gland hyperplasia

Epithelial injury, oedema

Smooth muscle cell proliferation

- Bronchoconstriction

- Hyperreactivity
Distal airspace and vasculature

- Decreased alveolarization, vascular growth

- Abnormal vascular remodelling, tone and reactivity

- Impaired lymphatic function and structure

\section{Other}

- Control of breathing

- Sleep disordered

breathing

- Chest wall stability

- Diaphragmatic function budesonide reduced the rate of $\mathrm{BPD}$, a follow-up study showed no improvement in rate of death or disability and an increased risk of mortality compared with placebo. In a randomized controlled trial of 265 infants weighing $<1,500 \mathrm{~g}$ who were treated with combination budesonide and surfactant (Survanta) or surfactant alone administered intratracheally, rates of death or BPD were $66 \%$ with surfactant alone and $42 \%$ with the combination therapy ${ }^{191}$. Follow-up into infancy revealed no differences in rates of neurodevelopmental impairment. Although promising, uncertainty about the optimal dose persists ${ }^{192}$ and this intervention needs to be tested further in larger, multicentre trials.

Inhaled nitric oxide. Inhaled nitric oxide (iNO) is an effective, widely used treatment for neonatal pulmonary hypertension. Studies in animal models showed that NO is required for normal lung development and that replacement therapy may benefit the injured lung ${ }^{193,194}$. However, these benefits have not been replicated in human infants. The authors of an IPD meta-analysis that included 14 randomized controlled trials and 3,430 preterm infants suggested that routine use of iNO in this population could not be recommended ${ }^{195}$. Observations that the effectiveness of iNO might vary by ethnicity led to an IPD meta-analysis examining this question. A pooled analysis of three randomized controlled trials showed an interaction between ethnicity and the outcome death or BPD. A reduction in rate of this outcome was found in the African American population but not the white or Hispanic groups ${ }^{196}$. This observation may be regarded as hypothesis-generating and an appropriate basis for further prospective randomized controlled trials ${ }^{197}$.

Oxygen saturation targeting. Balancing the risks and benefits of oxygen therapy has been a long-term, unrealized goal of neonatal intensive care. Currently, oxygen administration to preterm infants is titrated to achieve certain oxygen saturation ranges. An IPD meta-analysis of five high-quality randomized controlled trials comparing high (91-95\%) and low (85-89\%) saturation ranges provides useful guidance for clinicians ${ }^{198}$. The higher range was associated with a reduced risk of death and necrotizing enterocolitis but no difference in the primary outcome, the combined rate of death or major disability at a corrected age of 18-24 months. Treatment for retinopathy of prematurity was more common in infants in the high than in the low saturation target group but there was no difference in the rates of blindness. Importantly, there was no important difference in the rates of BPD between the two treatment groups.

\section{Long-term management}

Despite advances in early respiratory care to minimize ventilator-induced lung injury from delivery to later times, some preterm infants develop severe chronic respiratory disease and related comorbidities that persist throughout their NICU course and after NICU discharge. Prolonged care needs include tracheostomy with mechanical ventilation, high $\mathrm{FiO}_{2}$ concentrations and multiple respiratory medications, such as 

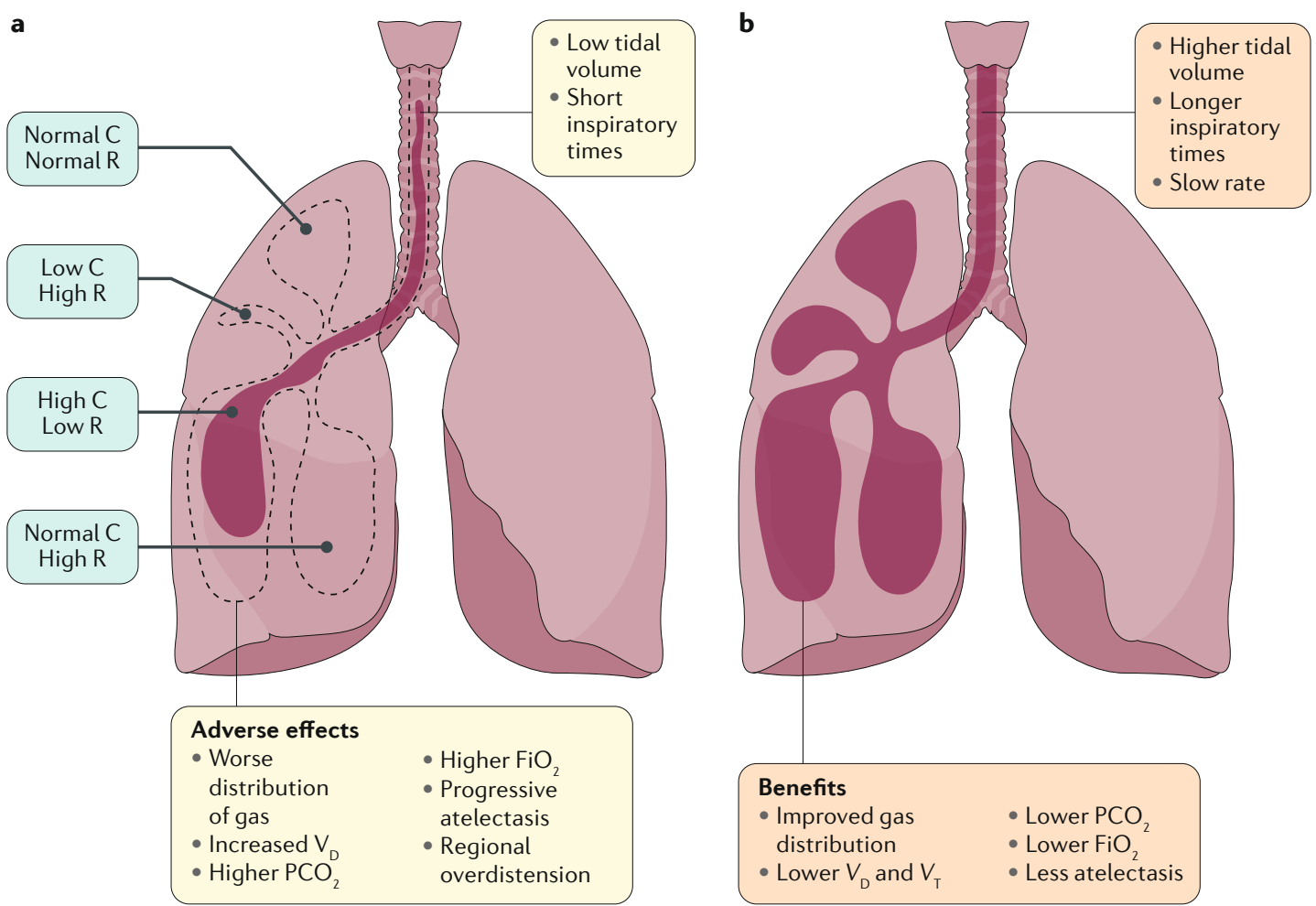

Fig. 7 | Alternative ventilation strategies in the treatment of heterogeneous lung disease in severe BPD. Schematic of the physiological effects of small-volume, rapid-rate ventilator support (panel a) and larger tidal volume, prolonged inspiratory time strategies (panel $\mathbf{b}$ ) in a lung that contains regional heterogeneity in the severity of lung disease, as is observed in infants with severe bronchopulmonary dysplasia (BPD). a | Small tidal volume breaths probably increase dead space ventilation, leading to atelectasis, hypercapnia and high oxygen requirements. $\mathbf{b} \mid$ Increased tidal volumes and inspiratory times may enhance the distribution of gas, leading to lower oxygen requirements, improved ventilation and less atelectasis. Compliance $(\mathrm{C})$ is a measure of the lung's ability to stretch and expand (distensibility of elastic tissue). Resistance $(\mathrm{R})$ is the resistance of the respiratory tract to airflow during inhalation and expiration. $\mathrm{FiO}_{2}$, fraction of inspired oxygen; $\mathrm{PCO}_{2}$, partial pressure of carbon dioxide; $\mathrm{V}_{\mathrm{D}}$, dead-space ventilation; $\mathrm{V}_{\mathrm{T}}$, tidal ventilation. Adapted with permission from REF. ${ }^{200}$, Elsevier.

intermittent corticosteroids, bronchodilators, diuretics and other drugs.

Preterm infants who require prolonged respiratory support at 36 weeks corrected age, including the need for tracheostomy and chronic ventilator care, constitute the most severe subgroup of BPD by NIH criteria (defined as type 2 severe BPD; TABLE 1). These infants are at the highest risk of late mortality, poor cardiopulmonary outcomes and extensive comorbidities, including worse somatic growth, neurocognitive problems and developmental delays ${ }^{115}$. Management is further complicated by the diverse contributors to the underlying lung pathophysiology, including airways, airspace and vascular components that probably define distinct 'respiratory

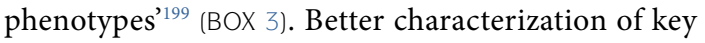
physiological contributions to pathophysiology in individual patients with severe BPD will probably enhance overall management and result in more selective and targeted use of pharmacological agents for therapy.

The goals and challenges in the management of cardiopulmonary dysfunction in the setting of the complex problems associated with severe BPD are strikingly different from those primarily focused on disease prevention and short-term therapies ${ }^{15,116,199-201}$. Despite some consensus recommendations ${ }^{115,200}$, striking variations exist in respiratory care, diagnostic evaluations, selection and use of drug therapies, patterns of long-term follow-up and other factors that reflect many differences among centres and among practitioners ${ }^{116}$. In the absence of data from multicentre randomized controlled trials, current approaches for the long-term management of severe BPD remain primarily based on clinical experience, physiology-based strategies and small case series. Thus, optimal management strategies to enhance long-term outcomes in patients with severe BPD remain uncertain.

In addition to the absence of proven and effective evidence-based strategies to improve outcomes, patients with chronic ventilator-dependent BPD have historically been cared for in acute care settings. Management strategies for chronic disease differ considerably from those for acute respiratory failure, especially regarding approaches to mechanical ventilation ${ }^{200}$ (FIG. 7). When compared with approaches for acute respiratory failure, neonatologists may have less experience with ventilator management of infants with severe BPD and the different approaches to optimize care. Most importantly, in the setting of chronic complex lung disease, poor communication can exist among providers, subspecialists, nursing staff and families during prolonged hospitalization, which can lead to inconsistent care and adverse 
outcomes. High staff turnover and infrequent discussions among doctors and bedside staff and between parents and the medical team may contribute to these inconsistencies. Interdisciplinary care teams have the potential to alleviate many of these issues and improve outcomes in these infants. The formation of such teams has been associated with improved survival ${ }^{202}$, shorter hospitalization and reduced costs ${ }^{203}$. The clinical course in infants with severe BPD is complex, with multiple morbidities, including frequent hospitalization throughout childhood, and often with poor continuity of care ${ }^{115,199}$. As a result, linking in-patient and longterm out-patient care is important for enhancing the continuity of care, understanding the complex clinical course, and anticipating complications due to persistent morbidities and other issues ${ }^{115}$.

The developing lung has remarkable capacity for remodelling, yet disease of the airways, airspace and vasculature can persist (FIG. 8a). Multiple studies have
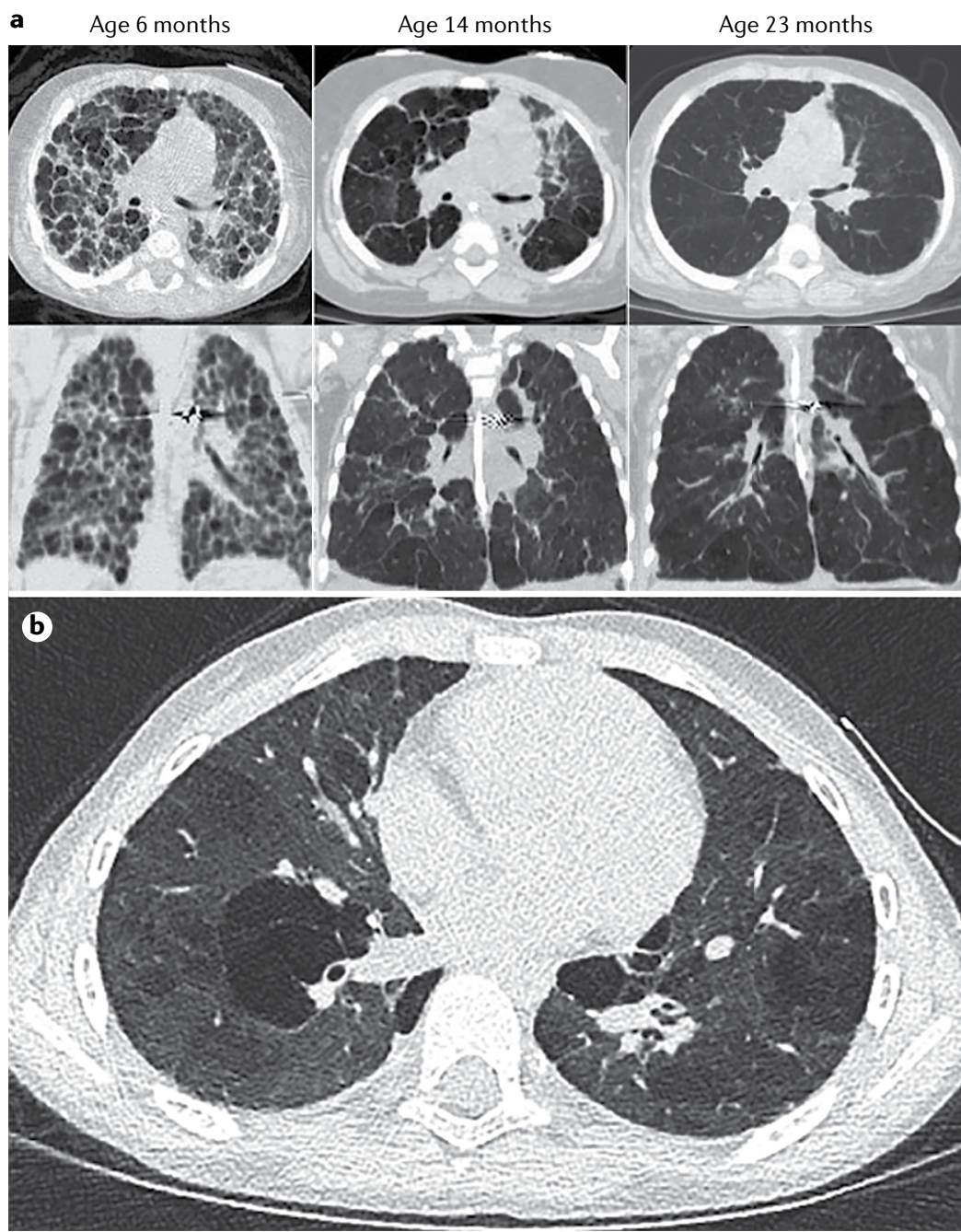

Fig. 8 | Structural changes in the lungs in severe BPD. a | Serial high-resolution CT scans illustrate time-related changes in lung architecture with regional heterogeneity in an infant with severe bronchopulmonary dysplasia (BPD). Extensive remodelling occurs over time, but persistent lung hyperinflation with abnormal lung parenchyma persists at 23 months. $\mathbf{b}$ | Chest CT scan of an 8-year-old girl who was born at 26 weeks gestation and had a history of severe bronchopulmonary dysplasia and pulmonary hypertension. Part a reproduced with permission from REF. ${ }^{200}$, Elsevier. shown abnormalities in lung function and structure in patients with BPD that persist throughout childhood, adolescence and young adulthood. In addition, several studies have further emphasized that prematurely born young adults with or without a history of BPD are at risk of PVD and abnormal cardiac performance ${ }^{204-210}$. These multiple reports suggest that there is a high risk of severe respiratory impairment after an apparent 'honeymoon' period during childhood and highlight the vital importance of early interventions, linking early NICU care with close follow-up in the ambulatory setting over time, and further strengthening collaborations between paediatric and adult subspecialists, especially in the fields of pulmonary medicine and cardiology. More research is clearly needed to determine optimal strategies to improve lung and cardiovascular health across the lifespan in preterm infants with and without severe $\mathrm{BPD}$ to ultimately improve quality of life (QOL).

\section{Quality of life}

Premature infants with BPD have a longer initial hospitalization than those without $\mathrm{BPD}^{211}$, and $\mathrm{BPD}$ remains a substantial life-long burden. The costs of the disorder are both social and economic and are measured in impaired childhood health and QOL, family stress, economic hardship and increased health-care costs ${ }^{212-214}$.

\section{Support for parents}

Mothers of very preterm infants experience substantially more psychological distress in the first months of life than mothers of full-term infants. This stress may continue for the first few years beyond discharge from the NICU, particularly for parents of the highestrisk infants ${ }^{215}$. As infants with severe BPD reach termequivalent gestation, their parents are often asked whether they prefer oxygen and home monitoring or a more prolonged hospital stay. At least half of parents express fear over home oxygen while still in the hospital, although by 3 months, most parents seem to adapt, and prefer this option to facilitate earlier discharge to home ${ }^{216}$. The stress of caring for a baby with severe BPD extends well beyond the first few months of life. In one parental survey, parental perceived health-related QOL of children with severe BPD at 18-36 months corrected age was substantially lower than that of children born at term, and also lower than that of preterm children without $\mathrm{BPD}^{217}$. Parents vividly described living in fear of their baby catching a cold, being rehospitalized, or dying from BPD, and considered that they were not provided with enough support and education to meet their needs ${ }^{218}$.

\section{Respiratory outcomes}

Up to $50 \%$ of children with BPD are rehospitalized during the first 2 years of life ${ }^{219,220}$. Respiratory viruses, such as respiratory syncytial virus, influenza virus and rhinovirus, frequently precipitate pulmonary exacerbations, increasing the risk of hospitalization in this age $\operatorname{group}^{221-223}$.

Lung function, measured as FEV1, has improved in preterm infants over the past few decades ${ }^{224}$. Nevertheless, children born preterm with and without BPD 

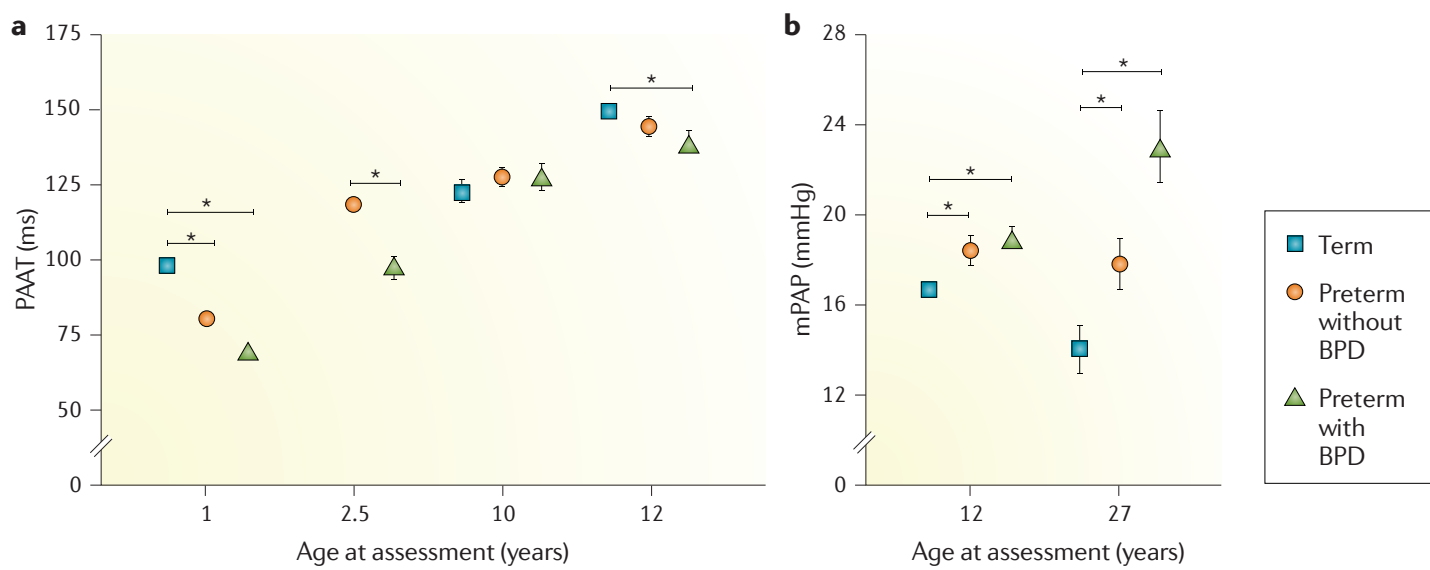

Fig. 9 | Persistent subclinical pulmonary vascular disease in BPD. a |After extreme preterm birth (average gestational age of 27 weeks), several echocardiography-based studies demonstrate lower pulmonary artery acceleration time (PAAT) beyond infancy into early adolescence, suggesting higher pulmonary vascular pressure and resistance ${ }^{210,247-249} \cdot \mathbf{b} \mid$ Mean pulmonary artery pressure (mPAP) is consistently highest during adolescence and early adulthood among those who were preterm infants with bronchopulmonary dysplasia (BPD) ${ }^{205,210} .{ }^{*} P<0.05$ by two-way ANOVA with Sidak's multiple comparisons test.

continue to have lower lung function than children born at term ${ }^{225,226}$. Children with BPD have a higher risk of having asthma-like symptoms during pre-school and school-age years ${ }^{199,227-230}$. Symptoms of wheezing, cough, airway reactivity and shortness of breath are frequently encountered in this population, along with exerciseinduced expiratory flow limitation ${ }^{228,231,232}$. Impaired postnatal alveolar growth may also occur in children with BPD. A study in young children with BPD found normal alveolar volumes but decreased pulmonary diffusion capacity, consistent with impaired alveolar growth compared with children born at term ${ }^{233}$. However, another study in school-age children born extremely prematurely showed similar alveolar sizes as in term and mildly preterm infants, suggesting the capacity for catchup alveolar growth ${ }^{234}$. Nonetheless, the extremely preterm children in this study had lower FEV1 values than term and mildly preterm children. Although obstructive lung disease is more commonly found in children with $\mathrm{BPD}$ than in those without BPD, restrictive lung disease can also be present ${ }^{235}$. Factors contributing to chronic lung dysfunction in children with BPD can include non-synchronous increases in lung size and airway calibre (contributing to airflow obstruction), chronic airway inflammation, air trapping and emphysematous changes $^{230,236-240}$ (FIC. 8b).

Several studies have shown a decline in lung function in preterm children as they age $\mathrm{a}^{236,241}$. As an association exists between reduced lung function in early life and chronic obstructive pulmonary disease (COPD) in adult life, there is a concern that children with BPD are at increased risk of early-onset $\mathrm{COPD}^{242}$. Environmental exposures, including in utero and postnatal tobacco smoke exposure, may adversely affect lung function in children with $\mathrm{BPD}^{30}$. In addition, genetic ${ }^{31,243}$ and epigenetic factors ${ }^{29}$ may influence severity of respiratory symptoms and long-term outcomes in children with BPD. Risk stratification guidelines that include gestational age, lung function, and genetic and environmental factors are needed to enable identification of preterm children at increased risk of respiratory symptoms and decline during childhood and adult life.

\section{Pulmonary vascular disease}

Although numerous studies have addressed the shortterm and long-term effects of BPD on respiratory QOL, few if any have accounted for the additive effects of PVD. Although not specific to BPD, QOL surveys of parents of children with pulmonary hypertension who were treated with pulmonary vasodilators have found substantially lower QOL scores and higher parental stress, with scores even worse than those reported in children with cancer or congenital heart disease $\mathrm{e}^{24,245}$. Limited data indicate that treatment of BPD-related pulmonary hypertension correlates with improvements in functional status and thus presumably $\mathrm{QOL}^{246}$. Many of these infants are able to wean from vasodilator therapy, although the long-term effect of early PVD remains largely undefined.

There is growing evidence that premature infants with or without BPD who escape the neonatal window without developing clinical PVD have subclinical PVD that persists into adolescence and adulthood (FIG. 9). Echocardiographic markers, such as pulmonary artery acceleration time, indicate the presence of subtle PVD through childhood in preterm infants ${ }^{210,247-249}$. By adolescence, mild elevations in mean pulmonary artery pressure are detected, which are highest in pretermborn adults with $\mathrm{BPD}^{210}$. By right heart catheterization, adults who were born moderately to extremely preterm also have higher mean pulmonary artery pressure than those born at term ${ }^{205}$. Although most individuals in these studies fell below treatment thresholds, numerous studies now associate subclinical elevations in mean pulmonary artery pressure with increased overall mortality ${ }^{250-253}$. Finally, right ventricular function is a primary driver of overall function and QOL in pretermborn adults with PVD. Limited evidence demonstrates right ventricular dysfunction in preterm-born adults despite subclinical PVD, and thus potential for persistent impairment in $\mathrm{QOL}^{205,208}$. 


\section{Box 4 | Priority topics to advance the understanding and prevention of BPD}

\section{Consensus definition of bronchopulmonary dysplasia (BPD)}

- Definition that is based on pathophysiology and not on components of care (such as pressure and oxygen)

- Predict 'long-term' pulmonary and health-care outcomes

- Definition of BPD phenotypes and endotypes

- Useful as a clinical trial end point

Mechanistic understanding of normal lung development, injury and repair and clinical translation

- Capitalize on current cutting-edge single-cell technology and systems biology to understand the molecular pathways and cell-specific interactions in lung development and injury

- Develop and optimize the use of better animal models that integrate the multiple mechanisms that contribute to BPD pathogenesis

\section{Biomarkers}

- Capitalize on the parallel development of 'omics' and systems biology to identify preterm infants for targeted early interventions to minimize injury and promote repair

- Development of novel non-invasive technologies for longitudinal assessment of lung function and imaging

- Combine clinical, functional, imaging and biological markers to better guide care and interventions

Effective interventions that meaningfully reduce incidence and severity of BPD

- Cell-based therapies: improve understanding of the biology of repair cells, identify reliable potency assays and fine-tune the manufacturing process

- Identify new therapeutic targets in injury and repair pathways (for example, insulinlike growth factor 1, IL-1 receptor antagonist and modulators of the NOD-, LRR- and pyrin domain-containing 3 (NLRP3) inflammasome)

- Facilitate clinical translation of preclinical research. For example, enhance the rigour of preclinical research in testing promising therapies to accelerate safe and efficient clinical translation
Both early use $\mathrm{e}^{259}$ and later use ${ }^{260}$ of corticosteroids substantially decrease the risk of BPD. However, research on corticosteroid use has stalled because of concerns about long-term developmental outcomes in treated infants. In particular, the hope that hydrocortisone would be a safer alternative to high-dose dexamethasone has not fully born fruit. Hydrocortisone may or may not be as effective as dexamethasone, and few trials have demonstrated that it is truly safer. However, the decreasing use of steroids in the past decade, to a baseline of $8 \%$ in VLBW infants ${ }^{24}$, is laudable. Whereas caffeine administration seems to decrease the risk of BPD in various at-risk populations ${ }^{174}$, other pharmacological therapies, such as vitamin $\mathrm{A}$, have not found widespread $\mathrm{use}^{261}$. The debate about the role of iNO in both the prevention and the treatment of evolving BPD continues, but little evidence exists to support a meaningful impact of this treatment ${ }^{262}$.

The future of clinical care of BPD will undoubtedly rely on a greater understanding of the 'clinical phenotype' of infants with BPD; that is, understanding the contribution of airway disease, parenchymal lung disease and PVD to the respiratory difficulty ${ }^{3}$. A greater appreciation of the vast difference between the underlying pulmonary vascular pathology of evolving or established BPD and the pathophysiology of prematurity in the newly born preterm infant is essential to the establishment of effective approaches to the care of these infants. Refinements in BPD definitions will clearly help in the identification of infants who are at higher risk of BPD (and thus might benefit from future trials of either available therapies, such as corticosteroids, or novel therapies that are yet to be tested) and give a greater understanding of the extent of improvement (or lack of improvement) that is associated with various interventions. However, existing definitions (such as supplemental oxygen at 36 weeks PMA) predict poor outcome and continue to be useful in clinical practice to decrease the prevalence of BPD in NICUs ${ }^{14}$ (see list of priorities in BOX 4).

Perhaps through improved quality of care and by enhancing each of these small benefits, we might see continued improvement in outcomes by reducing the incidence and severity of $\mathrm{BPD}^{263}$. Top performing neonatal units maintain rates of BPD as low as $20 \%{ }^{254}$. For the future, we will have to look to these incremental improvements, as well as breakthrough therapies, such as mesenchymal stromal cells (MSCs), to truly address this problem.

\section{Emerging treatments}

Within less than 10 years from the initial proof of concept studies ${ }^{264,265}$, promising preclinical data with MSCs $^{266}$ have lead to phase I clinical trials. Two trials have shown that administration of a single intratracheal dose of allogeneic cord blood-derived MSCs in extreme preterm infants during the first week of life is feasible and without severe adverse events attributable to the intervention $^{267,268}$. Similarly, the successful off-label use of repeated intravenous injections of bone marrow-derived MSCs in two extreme preterm infants with severe and advanced BPD supports the feasibility of this therapy ${ }^{269}$. Ongoing phase II trials will indicate whether MSC 
therapy is safe and effective. Numerous other types of repair cells are being investigated in the clinic ${ }^{270}$ and in the laboratory for use in BPD therapy. Despite rapid clinical translation, cell-based therapies for regenerative indications are still in their infancy and major knowledge gaps remain. A better understanding of the biology and mechanism of action of MSCs and other repair cell types $^{271-273}$ and the link between resident stem or progenitor cells and disease pathogenesis ${ }^{274-277}$, together with technological advances, will allow the development of potency assays and manufacturing processes ${ }^{278}$ to yield superior cell products that may truly curb complications of prematurity and improve outcomes.

Other anti-inflammatory agents, such as IL-1 receptor antagonist (IL-1Ra) or modulators of the NOD-, LRRand pyrin domain-containing 3 (NLRP3) inflammasome upstream of IL- $1 \beta$, seem to be encouraging in preclinical studies (reviewed in $\mathrm{REF}^{279}$ ). For example, recombinant human insulin-like growth factor 1 complexed with its binding protein (rhIGF1-rhIGFBP3) was investigated in a phase II trial for the prevention of retinopathy of prematurity in 121 extreme preterm infant $\mathrm{s}^{280}$. Although development of retinopathy of prematurity was unaltered, rhIGF1-rhIGFBP3 treatment decreased the occurrence of severe BPD. These results warrant a trial focused on BPD as the primary end point.

\section{Expected benefits of lung research}

Technical advances in imaging, transcriptomics, epigenetics and lineage labelling are providing ever more detailed insights into the diverse cell types, including stem or progenitor cells, and the molecular processes by which cells interact to form and regenerate the lung after injury ${ }^{53-55,281-283}$. Identification of the signalling and transcriptional networks that direct lung formation, repair and function might lead to the identification of pertinent biomarkers and inform clinical strategies to protect the critical progenitor cells and processes on which lung formation and repair depend. These insights will enable development of therapeutics aimed at enhancing normal tissue regeneration while inhibiting the cellular and molecular processes that underlie pathological tissue remodelling and loss of tissue that underlies many chronic lung diseases. In spite of severe lung dysfunction and injury associated with preterm birth, most infants with BPD achieve remarkable recovery of lung structure and function. Understanding the resilience of the neonatal lung will provide further insights that will be highly relevant to the processes of normal and pathological repair associated with chronic lung diseases that are active throughout the lifespan.

Published online: 14 November 2019
1. Northway, W. H. Jr., Rosan, R. C. \& Porter, D. Y. Pulmonary disease following respirator therapy of hyaline-membrane disease. Bronchopulmonary dysplasia. N. Engl. J. Med. 276, 357-368 (1967). This paper provides the first description of BPD, and many of the findings are true today.

2. Abman, S. H., Bancalari, E. \& Jobe, A. The evolution of bronchopulmonary dysplasia after 50 years. Am. J. Respir. Crit. Care Med. 195, 421-424 (2017).

3. Higgins, R. D. et al. Bronchopulmonary dysplasia: executive summary of a workshop. J. Pediatr. 197. 300-308 (2018)

4. Goldenberg, R. L., Culhane, J. F., lams, J. D. $\&$ Romero, R. Epidemiology and causes of preterm birth. Lancet 371, 75-84 (2008)

5. Narayanan, M. et al. Alveolarization continues during childhood and adolescence: new evidence from helium-3 magnetic resonance. Am. J. Respir. Crit. Care Med. 185, 186-191 (2012).

6. Zeitlin, J. et al. Preterm birth time trends in Europe: a study of 19 countries. BJOG 120, 1356-1365 (2013).

7. Ferre, C., Callaghan, W., Olson, C., Sharma, A. \& Barfield, W. Effects of maternal age and age-specific preterm birth rates on overall preterm birth rates United States, 2007 and 2014. MMWR Morb. Mortal Wkly. Rep. 65, 1181-1184 (2016).

8. Stoll, B. J. et al. Neonatal outcomes of extremely preterm infants from the NICHD Neonatal Research Network. Pediatrics 126, 443-456 (2010).

9. Wadhawan, R. et al. Does labor influence neonatal and neurodevelopmental outcomes of extremely-low-birthweight infants who are born by cesarean delivery? Am. J. Obstet. Gynecol. 189, 501-506 (2003).

10. Lapcharoensap, W. et al. Hospital variation and risk factors for bronchopulmonary dysplasia in a population-based cohort. JAMA Pediatr. 169 e 143676 (2015)

11. Adams, M. et al. Variability of very low birth weight infant outcome and practice in Swiss and US neonatal units. Pediatrics 141, e20173436 (2018).

12. Bhunwal, S., Mukhopadhyay, K., Bhattacharya, S Dey, P. \& Dhaliwal, L. K. Bronchopulmonary dysplasia in preterm neonates in a level III neonatal unit in India. Indian Pediatr. 55, 211-215 (2018)

13. Bose, C. et al. Fetal growth restriction and chronic lung disease among infants born before the 28th week of gestation. Pediatrics 124, e450-e458 (2009).

14. Isayama, T. et al. Revisiting the definition of bronchopulmonary dysplasia: effect of changing panoply of respiratory support for preterm neonates. JAMA Pediatr. 171, 271-279 (2017).

These data from the Canadian Neonatal Network identified the use of oxygen and/or respiratory support as a better indicator of chronic respiratory insufficiency than oxygen alone, and that assessment at term equivalent ( 40 weeks postmenstrual age) increases the predictive value.

15. Lee, J. H., Noh, O. K., Chang, Y. S. \& Korean Neonatal Network. Neonatal outcomes of very low birth weight infants in Korean Neonatal Network from 2013 to 2016. J. Korean Med. Sci. 34, e40 (2019).

16. Lin, H. J. et al. Mortality and morbidity of extremely low birth weight infants in the mainland of China: a multi-center study. Chin. Med. J. 128, 2743-2750 (2015).

17. Su, B. H. et al. Neonatal outcomes of extremely preterm infants from Taiwan: comparison with Canada, Japan, and the USA. Pediatr. Neonatol. 56 46-52 (2015)

18. Shah, P. S. et al. Neonatal outcomes of very low birth weight and very preterm neonates: an international comparison. J. Pediatr. 177, 144-152 e146 (2016).

19. Ambalavanan, N. et al. Predictors of death or bronchopulmonary dysplasia in preterm infants with respiratory failure. J. Perinatol. 28, 420-426 (2008)

20. Lemons, J. A. et al. Very low birth weight outcomes of the National Institute of Child Health and Human Development Neonatal Research Network, January 1995 through December 1996. Pediatrics 107, e1 (2001).

21. Marshall, D. D. et al. Risk factors for chronic lung disease in the surfactant era: a North Carolina population-based study of very low birth weight infants. North Carolina Neonatologists Association. Pediatrics 104, 1345-1350 (1999).

22. Oh, W. et al. Association between fluid intake and weight loss during the first ten days of life and risk of bronchopulmonary dysplasia in extremely low birth weight infants. J. Pediatr. 147, 786-790 (2005).

23. Rojas, M. A. et al. Changing trends in the epidemiology and pathogenesis of neonatal chronic lung disease. J. Pediatr. 126, 605-610 (1995).

24. Stoll, B. J. et al. Trends in care practices, morbidity, and mortality of extremely preterm neonates, 1993-2012. JAMA 314, 1039-1051 (2015).

25. Young, T. E., Kruyer, L. S., Marshall, D. D. \& Bose, C. L Population-based study of chronic lung disease in very low birth weight infants in North Carolina in 1994 with comparisons with 1984. The North Carolina Neonatologists Association. Pediatrics 104, e17 (1999).
26. Younge, N. et al. Survival and neurodevelopmental outcomes among periviable infants. N. Engl. J. Med. 376, 617-628 (2017)

27. Walsh, M. C. et al. Impact of a physiologic definition on bronchopulmonary dysplasia rates. Pediatrics $\mathbf{1 1 4}$ 1305-1311 (2004).

28. Hartling, L., Liang, Y \& Lacaze-Masmonteil, T. Chorioamnionitis as a risk factor for bronchopulmonary dysplasia: a systematic review and meta-analysis. Arch. Dis. Child Fetal. Neonatal Ed. 97, F8-F17 (2012).

29. McEvoy, C. T. \& Spindel, E. R. Pulmonary effects of maternal smoking on the fetus and child: effects on lung development, respiratory morbidities, and life long lung health. Paediatr. Respir. Rev. 21, 27-33 (2017).

30. Morrow, L. A. et al. Antenatal determinants of bronchopulmonary dysplasia and late respiratory disease in preterm infants. Am. J. Respir. Crit. Care Med. 196, 364-374 (2017).

31. Lavoie, P. M., Pham, C. \& Jang, K. L. Heritability of bronchopulmonary dysplasia, defined according to the consensus statement of the National Institutes of Health. Pediatrics 122, 479-485 (2008).

32. Parker, R. A., Lindstrom, D. P. \& Cotton, R. B. Evidence from twin study implies possible genetic susceptibility to bronchopulmonary dysplasia. Semin. Perinatol. 20, 206-209 (1996).

33. Bhandari, V. et al. Genetics of bronchopulmonary dysplasia: when things do not match up, it is only the beginning. J. Pediatr. 208, 298-299 (2019).

34. Lal, C. V., Bhandari, V. \& Ambalavanan, N. Genomics, microbiomics, proteomics, and metabolomics in bronchopulmonary dysplasia. Semin. Perinatol. 42 425-431 (2018).

35. Parad, R. B. et al. Role of genetic susceptibility in the development of bronchopulmonary dysplasia. J. Pediatr. 203, 234-241.e2 (2018).

36. Torgerson, D. G. et al. Ancestry and genetic associations with bronchopulmonary dysplasia in preterm infants. Am. J. Physiol. Lung Cell. Mol. Physiol. 315, L858-L869 (2018).

37. Yu, K. H., Li, J., Snyder, M., Shaw, G. M. $\delta$ O'Brodovich, H. M. The genetic predisposition to bronchopulmonary dysplasia. Curr. Opin. Pediatr. 28 318-323 (2016)

38. Ryan, S. W., Nycyk, J. \& Shaw, B. N. Prediction of chronic neonatal lung disease on day 4 of life. Eur. J. Pediatr. 155, 668-671 (1996).

39. Laughon, M. et al. Antecedents of chronic lung disease following three patterns of early respiratory 
disease in preterm infants. Arch. Dis. Child Feta Neonatal Ed. 96, F114-F120 (2011).

40. Charafeddine, L., D'Angio, C. T. \& Phelps, D. L. Atypical chronic lung disease patterns in neonates. Pediatrics 103, 759-765 (1999).

41. Panickar, J., Scholefield, H., Kumar, Y., Pilling, D. W. $\&$ Subhedar, N. V. Atypical chronic lung disease in preterm infants. J. Perinat. Med. 32, 162-167 (2004).

42. Choi, C. W., Kim, B. I., Koh, Y. Y., Choi, J. H. \& Choi, J. Y. Clinical characteristics of chronic lung disease without preceding respiratory distress syndrome in preterm infants. Pediatr. Int. 47, 72-79 (2005).

43. Choi, C. W. et al. Risk factors for the different types of chronic lung diseases of prematurity according to the preceding respiratory distress syndrome. Pediatr. Int. 47, 417-423 (2005)

44. Streubel, A. H., Donohue, P. K. \& Aucott, S. W. The epidemiology of atypical chronic lung disease in extremely low birth weight infants. J. Perinatol. 28, 141-148 (2008)

45. Nobile, S. et al. New insights on early patterns of respiratory disease among extremely low gestational age newborns. Neonatology 112 53-59 (2017)

46. Merrill, J. D. et al. Dysfunction of pulmonary surfactant in chronically ventilated premature infants. Pediatr. Res. 56, 918-926 (2004).

47. Laughon, M. et al. Patterns of blood protein concentrations of ELGANs classified by three patterns of respiratory disease in the first 2 postnatal weeks. Pediatr. Res. 70, 292-296 (2011).

48. Laughon, M. et al. Patterns of respiratory disease during the first 2 postnatal weeks in extremely premature infants. Pediatrics 123, 1124-1131 (2009). The authors used data from the NICHD Neonatal Research Network to develop a BPD prediction model by postnatal day, which can be used to provide information to families, for deciding when to use corticosteroids and as a preliminary measure of drug effectiveness for BPD treatment in clinical trials.

49. Laughon, M. M. et al. Prediction of bronchopulmonary dysplasia by postnatal age in extremely premature infants. Am. J. Respir. Crit. Care Med. 183 1715-1722 (2011)

50. Onland, W. et al. Clinical prediction models for bronchopulmonary dysplasia: a systematic review and external validation study. BMC Pediatr. 13, 207 (2013).

51. Leroy, S. et al. A time-based analysis of inflammation in infants at risk of bronchopulmonary dysplasia. J. Pediatr. 192, 60-65.e1 (2018)

52. Metzger, R. J., Klein, O. D., Martin, G. R. \& Krasnow, M. A. The branching programme of mouse lung development. Nature 453, 745-750 (2008).

53. Hogan, B. L. et al. Repair and regeneration of the respiratory system: complexity, plasticity, and mechanisms of lung stem cell function. Cell Stem Cell 15, 123-138 (2014)

54. Whitsett, J. A., Kalin, T. V., Xu, Y. \& Kalinichenko, V. V. Building and regenerating the lung cell by cell. Physiol. Rev. 99, 513-554 (2019).

55. Guo, M. et al. Single cell RNA analysis identifies cellular heterogeneity and adaptive responses of the lung at birth. Nat. Commun. 10, 37 (2019).

56. Whitsett, J. A., Wert, S. E. \& Weaver, T. E. Diseases of pulmonary surfactant homeostasis. Annu. Rev. Pathol. 10, 371-393 (2015)

57. Jobe, A. H. The new bronchopulmonary dysplasia Curr. Opin. Pediatr. 23, 167-172 (2011).

58. Higano, N. S. et al. Neonatal pulmonary magnetic resonance imaging of bronchopulmonary dysplasia predicts short-term clinical outcomes. Am. J. Respir. Crit. Care Med. 198, 1302-1311 (2018).

59. Surate Solaligue, D. E., Rodriguez-Castillo, J. A., Ahlbrecht, K. \& Morty, R. E. Recent advances in our understanding of the mechanisms of late lung development and bronchopulmonary dysplasia. Am. J. Physiol. Lung Cell Mol. Physiol. 313 L1101-L1153 (2017).

The authors extensively review the animal models that have contributed to an understanding of the lung injury in BPD, with an emphasis on transgenic mouse models.

60. Jobe, A. H. Animal models, learning lessons to prevent and treat neonatal chronic lung disease. Front. Med. 2, 49 (2015)

61. Morty, R. E. Recent advances in the pathogenesis of BPD. Semin. Perinatol. 42, 404-412 (2018).

62. Balany, J. \& Bhandari, V. Understanding the impact of infection, inflammation, and their persistence in the pathogenesis of bronchopulmonary dysplasia. Front. Med. 2, 90 (2015)

63. Lee, J. W. \& Davis, J. M. Future applications of antioxidants in premature infants. Curr. Opin. Pediatr. 23, 161-166 (2011)

64. Savani, R. C. Modulators of inflammation in bronchopulmonary dysplasia. Semin. Perinatol. 42, 459-470 (2018).

65. Onland, W., De Jaegere, A. P., Offringa, M. \& van Kaam, A. Systemic corticosteroid regimens for prevention of bronchopulmonary dysplasia in preterm infants. Cochrane Database Syst. Rev. 1, CD010941 (2017).

66. Pillow, J. J. et al. Bubble continuous positive airway pressure enhances lung volume and gas exchange in preterm lambs. Am. J. Respir. Crit. Care Med. 176, 63-69 (2007).

67. Kim, C. J. et al. Acute chorioamnionitis and funisitis: definition, pathologic features, and clinical significance. Am. J. Obstet. Gynecol. 213, S29-S52 (2015).

68. Jobe, A. H. \& Goldenberg, R. L. Antenatal corticosteroids: an assessment of anticipated benefits and potential risks. Am. J. Obstet. Gynecol. 219 62-74 (2018)

This extensive summary reviews the effects of antenatal corticosteroids and fetal exposure to inflammation on clinical lung maturation

69. Kallapur, S. G., Presicce, P., Rueda, C. M., Jobe, A. H. $\&$ Chougnet, C. A. Fetal immune response to chorioamnionitis. Semin. Reprod. Med. 32, 56-67 (2014).

70. Andrews, W. W. et al. The Alabama preterm birth study: polymorphonuclear and mononuclear cell placental infiltrations, other markers of inflammation, and outcomes in 23- to 32-week preterm newborn infants. Am. J. Obstet. Gynecol. 195, 803-808 (2006).

71. Goldenberg, R. L. et al. The Alabama preterm birth study: umbilical cord blood Ureaplasma urealyticum and Mycoplasma hominis cultures in very preterm newborn infants. Am. J. Obstet. Gynecol. 198, 43. e1-43.e5 (2008)

72. Viscardi, R. M. \& Kallapur, S. G. Role of Ureaplasma respiratory tract colonization in bronchopulmonary dysplasia pathogenesis: current concepts and update Clin. Perinatol. 42, 719-738 (2015).

73. Jobe, A. H. Effects of chorioamnionitis on the fetal lung. Clin. Perinatol. 39, 441-457 (2012).

74. Kallapur, S. G. et al. Chronic fetal exposure to Ureaplasma parvum suppresses innate immune responses in sheep. J. Immunol. 187, 2688-2695 (2011).

75. Gisslen, T. et al. Repeated exposure to intra-amniotic LPS partially protects against adverse effects of intravenous LPS in preterm lambs. Innate Immun. $\mathbf{2 0}$ 214-224 (2014).

76. Lal, C. V. et al. The airway microbiome at birth Sci. Rep. 6, 31023 (2016)

77. Pammi, M. et al. Airway microbiome and development of bronchopulmonary dysplasia in preterm infants: a systematic review. J. Pediatr. 204, 126-133.e2 (2019).

78. Fouron, J. C., Le Guennec, J. C., Villemant, D. Perreault, G. \& Davignon, A. Value of echocardiography in assessing the outcome of bronchopulmonary dysplasia of the newborn. Pediatrics 65, 529-535 (1980).

79. Khemani, E. et al. Pulmonary artery hypertension in formerly premature infants with bronchopulmonary dysplasia: clinical features and outcomes in the surfactant era. Pediatrics 120, 1260-1269 (2007).

80. Mourani, P. M. \& Abman, S. H. Pulmonary hypertension and vascular abnormalities in bronchopulmonary dysplasia. Clin. Perinatol. 42 , 839-855 (2015)

81. An, H. S. et al. Pulmonary hypertension in preterm infants with bronchopulmonary dysplasia. Korean Circ. J. 40, 131-136 (2010).

82. Bhat, R., Salas, A. A., Foster, C., Carlo, W. A. \& Ambalavanan, N. Prospective analysis of pulmonary hypertension in extremely low birth weight infants. Pediatrics 129, e682-e689 (2012).

83. Mourani, P. M. et al. Early pulmonary vascular disease in preterm infants at risk for bronchopulmonary dysplasia. Am. J. Respiratory Crit. Care Med. 191, 87-95 (2015)

84. Aikio, O., Metsola, J., Vuolteenaho, R., Perhomaa, M. \& Hallman, M. Transient defect in nitric oxide generation after rupture of fetal membranes and responsiveness to inhaled nitric oxide in very preterm infants with hypoxic respiratory failure. J. Pediatr. 161, 397-403.e1 (2012).
85. Chandrasekharan, P. et al. Early use of inhaled nitric oxide in preterm infants: is there a rationale for selective approach? Am. J. Perinatol. 34, 428-440 (2017).

86. Mirza, H. et al. Natural history of postnatal cardiopulmonary adaptation in infants born extremely preterm and risk for death or bronchopulmonary dysplasia. J. Pediatr. 198, 187-193 e181 (2018).

87. Giesinger, R. E. et al. Controversies in the identification and management of acute pulmonary hypertension in preterm neonates. Pediatr. Res. 82, 901-914 (2017).

88. Berenz, A., Vergales, J. E., Swanson, J. R. \& Sinkin, R. A Evidence of early pulmonary hypertension is associated with increased mortality in very low birth weight infants. Am. J. Perinatol. 34, 801-807 (2017).

89. Taglauer, E., Abman, S. H. \& Keller, R. L. Recent advances in antenatal factors predisposing to bronchopulmonary dysplasia. Semin. Perinatol. 42 , 413-424 (2018)

90. Abman, S. H. Bronchopulmonary dysplasia: "a vascular hypothesis". Am. J. Respir. Crit. Care Med. 164, 1755-1756 (2001)

91. Bhatt, A. J. et al. Disrupted pulmonary vasculature and decreased vascular endothelial growth factor, FIt-1, and TIE-2 in human infants dying with bronchopulmonary dysplasia. Am. J. Respir. Crit. Care Med. 164, 1971-1980 (2001).

92. Stenmark, K. R. \& Abman, S. H. Lung vascular development: implications for the pathogenesis of bronchopulmonary dysplasia. Annu. Rev. Physiol. 67 623-661 (2005)

93. Thebaud, B. \& Abman, S. H. Bronchopulmonary dysplasia: where have all the vessels gone? Roles of angiogenic growth factors in chronic lung disease. Am. J. Respir. Crit. Care Med. 175, 978-985 (2007).

94. Check, J. et al. Fetal growth restriction and pulmonary hypertension in premature infants with bronchopulmonary dysplasia. J. Perinatol. 33 , 553-557 (2013)

95. Mestan, K. K. et al. Placental pathologic changes of maternal vascular underperfusion in bronchopulmonary dysplasia and pulmonary

96. Keller, R. L. et al. Bronchopulmonary dysplasia and perinatal characteristics predict 1-year respiratory outcomes in newborns born at extremely low gestational age: a prospective cohort study. J. Pediatr. 187, 89-97.e3 (2017)

97. Baker, C. D. et al. Cord blood angiogenic progenitor cells are decreased in bronchopulmonary dysplasia. Eur. Respir. J. 40, 1516-1522 (2012).

98. Mestan, K. K. et al. Cord blood biomarkers of placental maternal vascular underperfusion predict bronchopulmonary dysplasia-associated pulmonary hypertension. J. Pediatr. 185, 33-41 (2017).

99. Voller, S. B. et al. Cord blood biomarkers of vascular endothelial growth (VEGF and sFIt-1) and postnatal growth: a preterm birth cohort study. Early Hum. Dev. 90, 195-200 (2014).

100. Mourani, P. M. et al. Early pulmonary vascular disease in preterm infants is associated with late respiratory outcomes in childhood. Am. J. Respir. Crit. Care Med. 199, 1020-1027 (2019).

This prospective study confirmed previous work in rodents showing that the development of PVD during the perinatal period increases the risk of BPD and pulmonary hypertension in human infants; early signs of PVD in preterm neonates on day 7 of life is associated with increased susceptibility to BPD and late respiratory disease, which requires more frequent emergency room visits, rehospitalization and respiratory medications throughout the first 2 years of life.

101. Mandell, E. W. \& Abman, S. H. Fetal vascular origins of bronchopulmonary dysplasia. J. Pediatr. $\mathbf{1 8 5}$, 7-10.e1 (2017)

102. Eriksson, L. et al. Perinatal conditions related to growth restriction and inflammation are associated with an increased risk of bronchopulmonary dysplasia. Acta Paediatr. 104, 259-263 (2015).

103. Hansen, A. R., Barnes, C. M., Folkman, J. \& McElrath, T. F. Maternal preeclampsia predicts the development of bronchopulmonary dysplasia. J. Pediatr. 156, 532-536 (2010).

104. Watterberg, K. L., Demers, L. M., Scott, S. M. $\&$ Murphy, S. Chorioamnionitis and early lung inflammation in infants in whom bronchopulmonary dysplasia develops. Pediatrics 97, 210-215 (1996). 
105. Steinhorn, R. et al. Chronic pulmonary insufficiency of prematurity: developing optimal endpoints for drug development. J. Pediatr. 191, 15-21.e1 (2017). This paper broadens the concept of chronic pulmonary insufficiency of prematurity (CPIP) to include chronic lung diseases in preterm infants that might be different from BPD, such as tracheomalacia or bronchomalacia. The paper emphasizes the importance of optimizing and harmonizing clinical definitions of CPIP, including BPD and later respiratory outcomes, while developing strong surrogate end points that are useful for regulators, industry, clinicians and families, to benefit future interventional trials and accelerate the development of new therapies for these high-risk and vulnerable patients.

106. Shennan, A. T., Dunn, M. S., Ohlsson, A., Lennox, K \& Hoskins, E. M. Abnormal pulmonary outcomes in premature infants: prediction from oxygen requirement in the neonatal period. Pediatrics 82 527-532 (1988)

107. Gage, S. et al. Determinants of chronic lung disease severity in the first year of life; a population based study. Pediatr. Pulmonol. 50, 878-888 (2015).

108. Jobe, A. H. \& Bancalari, E. Bronchopulmonary dysplasia. Am. J. Respir. Crit. Care Med. 163, 1723-1729 (2001).

109. Poindexter, B. B. et al. Comparisons and limitations of current definitions of bronchopulmonary dysplasia for the Prematurity and Respiratory Outcomes Program. Ann. Am. Thorac. Soc. 12, 1822-1830 (2015)

110. van Rossem, M. C. et al. Accuracy of the diagnosis of bronchopulmonary dysplasia in a referral-based health care system. J. Pediatr. 167, 540-544.e1 (2015).

111. Stoecklin, B., Simpson, S. J. \& Pillow, J. J. Bronchopulmonary dysplasia: rationale for a pathophysiological rather than treatment based approach to diagnosis. Paediatr. Respir. Rev. https:// doi.org/10.1016/j.prrv.2018.12.002 (2018)

112. Svedenkrans, J., Stoecklin, B., Jones, J. G., Doherty, D. A $\&$ Pillow, J. J. Physiology and predictors of impaired gas exchange in infants with bronchopulmonary dysplasia. Am. J. Respir. Crit. Care Med. 200 471-480 (2019)

113. Jensen, E. A. et al. The diagnosis of bronchopulmonary dysplasia in very preterm infants: an evidence-based approach. Am. J. Respir. Crit. Care Med. 200 751-759 (2019).

114. Ehrenkranz, R. A. et al. Validation of the National Institutes of Health consensus definition of bronchopulmonary dysplasia. Pediatrics 116 1353-1360 (2005).

115. Abman, S. H. et al. Interdisciplinary care of children with severe bronchopulmonary dysplasia. J. Pediatr. 181, 12-28.e1 (2017).

116. Krishnan, U. et al. Evaluation and management of pulmonary hypertension in children with bronchopulmonary dysplasia. J. Pediatr. 188 24-34.e1 (2017)

117. Jobe, A. H. \& Steinhorn, R. Can we define bronchopulmonary dysplasia? J. Pediatr. 188, 19-23 (2017).

118. Stark, A. R. et al. Adverse effects of early dexamethasone treatment in extremely-low-birthweight infants. N. Engl. J. Med. 344, 95-101 (2001).

119. Lal, C. V. \& Ambalavanan, N. Biomarkers, early diagnosis, and clinical predictors of bronchopulmonary dysplasia. Clin. Perinatol. 42, 739-754 (2015).

120. McEvoy, C. T. et al. Bronchopulmonary dysplasia: NHLBI workshop on the primary prevention of chronic lung diseases. Ann. Am. Thorac. Soc. 11, S146-S153 (2014).

This workshop report highlights promising areas of research to improve understanding of normal and aberrant lung development, to distinguish BPD endotypes and to identify biomarkers for more targeted therapeutic approaches to prevention

121. Aschner, J. L., Bancalari, E. H. \& McEvoy, C. T. Can we prevent bronchopulmonary dysplasia? J. Pediatr. 189 26-30 (2017)

122. Wai, K. C. et al. Early cumulative supplemental oxygen predicts bronchopulmonary dysplasia in high risk extremely low gestational age newborns. J. Pediatr. 177, 97-102.e2 (2016)

123. Alvarez-Fuente, M. et al. Exploring clinical, echocardiographic and molecular biomarkers to predict bronchopulmonary dysplasia. PLOS ONE 14 e0213210 (2019).

124. Mahlman, M. et al. Genes encoding vascular endothelial growth factor A (VEGF-A) and VEGF receptor 2 (VEGFR-2) and risk for bronchopulmonary dysplasia. Neonatology 108, 53-59 (2015).
125. Qi, Y., Jiang, Q., Chen, C., Cao, Y. \& Qian, L. Circulating endothelial progenitor cells decrease in infants with bronchopulmonary dysplasia and increase after inhaled nitric oxide. PLOS ONE 8, e79060 (2013)

126. De Paepe, M. E., Patel, C., Tsai, A., Gundavarapu, S. \& Mao, Q. Endoglin (CD105) up-regulation in pulmonary microvasculature of ventilated preterm infants. Am. J. Respir. Crit. Care Med. 178, 180-187 (2008).

127. Mohamed, W. A. W., Niyazy, W. H. \& Mahfouz, A. A Angiopoietin-1 and endostatin levels in cord plasma predict the development of bronchopulmonary dysplasia in preterm infants. J. Trop. Pediatr. 57, 385-388 (2010)

128. Janér, J., Andersson, S., Kajantie, E. \& Lassus, P. Endostatin concentration in cord plasma predicts the development of bronchopulmonary dysplasia in very low birth weight infants. Pediatrics 123, 1142-1146 (2009).

129. Vento, G. et al. Serum levels of seven cytokines in premature ventilated newborns: correlations with old and new forms of bronchopulmonary dysplasia. Intensive Care Med. 32, 723-730 (2006).

130. Tsao, P.-N. et al. Placenta growth factor elevation in the cord blood of premature neonates predicts poo pulmonary outcome. Pediatrics 113, 1348-1351 (2004).

131. Lal, C. V. \& Schwarz, M. A. Vascular mediators in chronic lung disease of infancy: role of endothelial monocyte activating polypeptide II (EMAP II). Birth Defects Res. A Clin. Mol. Teratol. 100, 180-188 (2014).

132. Ogihara, T. et al. Plasma KL-6 predicts the development and outcome of bronchopulmonary dysplasia. Pediatr. Res. 60, 613 (2006)

133. Fukunaga, S. et al. MMP-9 and TIMP-1 in the cord blood of premature infants developing BPD. Pediatr. Pulmonol. 44, 267-272 (2009).

134. Bhandari, A. \& Bhandari, V. Biomarkers in bronchopulmonary dysplasia. Paediatr. Respir. Rev. 14, 173-179 (2013)

135. Capoluongo, E. et al. Epithelial lining fluid neutrophilgelatinase-associated lipocalin levels in premature newborns with bronchopulmonary dysplasia and patency of ductus arteriosus. Int. J. Immunopathol. Pharmacol. 21, 173-179 (2008).

136. Aschner, J. L. et al. Challenges, priorities and novel therapies for hypoxemic respiratory failure and pulmonary hypertension in the neonate. J. Perinatol. 36, S32 (2016).

137. Ballard, P. L. et al. Inhaled nitric oxide increases urinary nitric oxide metabolites and cyclic guanosine monophosphate in premature infants: relationship to pulmonary outcome. Am. J. Perinatol. 32, 225-232 (2015).

138. Fike, C. D. \& Aschner, J. L. Looking beyond PPHN the unmet challenge of chronic progressive pulmonary hypertension in the newborn. Pulm. Circ. 3, 454-466 (2013).

139. Fike, C. D., Summar, M. \& Aschner, J. L. L-citrulline provides a novel strategy for treating chronic pulmonary hypertension in newborn infants. Acta Paediatr. 103, 1019-1026 (2014).

140. O'Connor, M. G. et al. Pulmonary hypertension in the premature infant population: analysis of echocardiographic findings and biomarkers. Pediatr. Pulmonol. 53, 302-309 (2018).

141. Perrone, S., Tataranno, M. \& Buonocore, C. Oxidative stress and bronchopulmonary dysplasia. J. Clin. Neonatol. 1, 109-114 (2012).

142. Saugstad, O. D. Oxidative stress in the newborn a 30-year perspective. Neonatology $\mathbf{8 8}, \mathbf{2 2 8 - 2 3 6}$ (2005).

143. Saugstad, O. D. Bronchopulmonary dysplasia and oxidative stress: are we closer to an understanding of the pathogenesis of BPD? Acta Paediatr. 86, 1277-1282 (1997).

144. Ballard P L et al Plasma biomarkers of oxidative stress: relationship to lung disease and inhaled nitric oxide therapy in premature infants. Pediatrics 121 555-561 (2008)

145. Ambalavanan, N. et al. Cytokines associated with bronchopulmonary dysplasia or death in extremely low birth weight infants. Pediatrics 123, 1132-1141 (2009).

146. Truog, W. E. et al. Inflammatory markers and mediators in tracheal fluid of premature infants treated with inhaled nitric oxide. Pediatrics 119 670-678 (2007)

147. Piersigilli, F. et al. Identification of new biomarkers of bronchopulmonary dysplasia using metabolomics. Metabolomics 15, 20 (2019).
148. Lal, C. V. et al. Early airway microbial metagenomic and metabolomic signatures are associated with development of severe bronchopulmonary dysplasia. Am. J. Physiol. Lung Cell. Mol. Physiol. 315, L810-L815 (2018).

149. Hamvas, A. et al. Exome sequencing identifies gene variants and networks associated with extreme respiratory outcomes following preterm birth. BMC Genet. 19, 94 (2018).

150. May, C. et al. Relation of exhaled nitric oxide levels to development of bronchopulmonary dysplasia. Arch. Dis. Child Fetal. Neonatal. Ed. 94, F205-F209 (2009).

151. Kim, G. B. Pulmonary hypertension in infants with bronchopulmonary dysplasia. Korean J. Pediatr. 53 688-693 (2010)

152. Day, C. L. \& Ryan, R. M. Bronchopulmonary dysplasia: new becomes old again! Pediatr. Res. 81, 210 (2016)

153. Zhang, Z.-O., Huang, X.-M. \& Lu, H. Early biomarkers as predictors for bronchopulmonary dysplasia in preterm infants: a systematic review. Eur. J. Pediatr. 173, 15-23 (2014).

154. Mahlman, M. et al. Genome-wide association study of bronchopulmonary dysplasia: a potential role for variants near the CRP gene. Sci. Rep. 7, 9271 (2017).

155. Tanay, A. \& Regev, A. Scaling single-cell genomics from phenomenology to mechanism. Nature 541 331-338 (2017)

156. Anderson, P. J. \& Doyle, L. W. Neurodevelopmental outcome of bronchopulmonary dysplasia. Semin. Perinatol. 30, 227-232 (2006).

157. Ratner, $\mathrm{V}$ et al. The contribution of intermittent hypoxemia to late neurological handicap in mice with hyperoxia-induced lung injury. Neonatology 92 50-58 (2007)

158. Cheong, J. L. Y. \& Doyle, L. W. An update on pulmonary and neurodevelopmental outcomes of bronchopulmonary dysplasia. Semin. Perinatol. 42, 478-484 (2018)

159. Committee on Fetus and Newborn. Respiratory support in preterm infants at birth. Pediatrics 133 171-174 (2014).

160. Schmolzer, G. M. et al. Non-invasive versus invasive respiratory support in preterm infants at birth: systematic review and meta-analysis. BMJ 347, f5980 (2013).

161. Lemyre, B., Davis, P. G., De Paoli, A. G. \& Kirpalani, H. Nasal intermittent positive pressure ventilation (NIPPV) versus nasal continuous positive airway pressure (NCPAP) for preterm neonates after extubation. Cochrane Database Syst. Rev. 2, CD003212 (2017).

162. Lemyre, B., Laughon, M., Bose, C. \& Davis, P. C. Early nasal intermittent positive pressure ventilation (NIPPV) versus early nasal continuous positive airway pressure (NCPAP) for preterm infants. Cochrane Database Syst. Rev. 12, CD005384 (2016).

163. Wilkinson, D., Andersen, C., O'Donnell, C. P., De Paoli, A. G. \& Manley, B. J. High flow nasal cannula for respiratory support in preterm infants. Cochrane Database Syst. Rev. 2, CD006405 (2016).

164. Seger, N. \& Soll, R. Animal derived surfactant extract for treatment of respiratory distress syndrome. Cochrane Database Syst. Rev. 8, CD007836 (2009).

165. Soll, R. F. Synthetic surfactant for respiratory distress syndrome in preterm infants. Cochrane Database Syst. Rev. 2, CD001149 (2000).

166. Kribs, A. et al. Nonintubated surfactant application vs conventional therapy in extremely preterm infants: a randomized clinical trial. JAMA Pediatr. 169 723-730 (2015)

167. Dargaville, P. A et al Minimally-invasive surfactant therapy in preterm infants on continuous positive airway pressure. Arch. Dis. Child Fetal Neonatal Ed. 98, F122-F126 (2013)

168. Aldana-Aguirre, J. C., Pinto, M., Featherstone, R. M $\&$ Kumar, M. Less invasive surfactant administration versus intubation for surfactant delivery in preterm infants with respiratory distress syndrome: a systematic review and meta-analysis. Arch. Dis. Child Fetal Neonatal. Ed. 102, F17-F23 (2017).

169. Dargaville, P. A. et al. The OPTIMIST-A trial: evaluation of minimally-invasive surfactant therapy in preterm infants 25-28 weeks gestation. BMC Pediatr. 14, 213 (2014)

170. Finer, N. N. et al. An open label, pilot study of Aerosurf(R) combined with nCPAP to prevent RDS in preterm neonates. J. Aerosol. Med. Pulm. Drug Deliv. 23, 303-309 (2010).

171. Roberts, K. D. et al. Laryngeal mask airway for surfactant administration in neonates: a randomized, controlled trial. J. Pediatr. 193, 40-46.e1 (2018). 
172. Ballard, R. A. et al. Randomized trial of late surfactant treatment in ventilated preterm infants receiving inhaled nitric oxide. J. Pediatr. 168, 23-29.e4 (2016).

173. Keller, R. L. et al. The randomized, controlled trial of late surfactant: effects on respiratory outcomes at 1 -year corrected age. J. Pediatr. 183, 19-25.e2 (2017)

174. Schmidt, B. et al. Caffeine therapy for apnea of prematurity. N. Engl. J. Med. 354, 2112-2121 (2006).

175. Schmidt, B. et al. Long-term effects of caffeine therapy for apnea of prematurity. N. Engl. J. Med. 357 1893-1902 (2007)

References 173 and 174 report results of the CAP trial in 2,000 infants and its long-term follow-up study, which convincingly demonstrated the pathway between shortened exposure to assisted ventilation, reduced rates of BPD and improved neurodevelopmental outcomes.

176. Roberts, D., Brown, J., Medley, N. \& Dalziel, S. R. Antenatal corticosteroids for accelerating fetal lung maturation for women at risk of preterm birth. Cochrane Database Syst. Rev. 3, CD004454 (2017).

177. Committee on Fetus and Newborn. Postnatal corticosteroids to treat or prevent chronic lung disease in preterm infants. Pediatrics 109, 330-338 (2002).

178. Doyle, L. W., Halliday, H. L., Ehrenkranz, R. A., Davis, P. G. \& Sinclair, J. C. An update on the impact of postnatal systemic corticosteroids on mortality and cerebral palsy in preterm infants: effect modification by risk of bronchopulmonary dysplasia. J. Pediatr. 165, 1258-1260 (2014).

179. Doyle, L. W. et al. Outcome at 2 years of age of infants from the DART study: a multicenter, international, randomized, controlled trial of low-dose dexamethasone. Pediatrics 119, 716-721 (2007)

180. Doyle, L. W. et al. Low-dose dexamethasone facilitates extubation among chronically ventilator-dependent infants: a multicenter, international, randomized, controlled trial. Pediatrics 117, 75-83 (2006).

181. Nuytten, A. et al. Evidence-based neonatal unit practices and determinants of postnatal corticosteroiduse in preterm births below 30 weeks GA in Europe. a population-based cohort study. PLOS ONE 12 , e0170234 (2017).

182. Watterberg, K. L. et al. Prophylaxis of early adrenal insufficiency to prevent bronchopulmonary dysplasia: a multicenter trial. Pediatrics 114, 1649-1657 (2004).

183. Watterberg, K. L., Gerdes, J. S., Gifford, K. L. \& Lin, H. M. Prophylaxis against early adrenal insufficiency to prevent chronic lung disease in premature infants. Pediatrics 104, 1258-1263 (1999).

184. Watterberg, K. L., Scott, S. M., Backstrom, C., Gifford, K. L. \& Cook, K. L. Links between early adrenal function and respiratory outcome in preterm infants: airway inflammation and patent ductus arteriosus. Pediatrics 105, 320-324 (2000).

185. Baud, O et al. Effect of early low-dose hydrocortisone on survival without bronchopulmonary dysplasia in extremely preterm infants (PREMILOC): a doubleblind, placebo-controlled, multicentre, randomised trial. Lancet 387, 1827-1836 (2016)

186. Baud, O. et al. Association between early low-dose hydrocortisone therapy in extremely preterm neonates and neurodevelopmental outcomes at 2 years of age. JAMA 317, 1329-1337 (2017).

187. Onland, W. et al. Effect of hydrocortisone therapy initiated 7 to 14 days after birth on mortality or bronchopulmonary dysplasia among very preterm infants receiving mechanical ventilation: a randomized clinical trial. JAMA 321, 354-363 (2019).

188. Shaffer, M. L. et al. Effect of prophylaxis for early adrenal insufficiency using low-dose hydrocortisone in very preterm infants: an individual patient data meta-analysis. J. Pediatr. 207, 136-142.e5 (2019).

189. Bassler, D. et al. Long-term effects of inhaled budesonide for bronchopulmonary dysplasia. N. Engl. J. Med. 378, 148-157 (2018).

190. Bassler, D. et al. Early inhaled budesonide for the prevention of bronchopulmonary dysplasia. N. Engl. J. Med. 373, 1497-1506 (2015).

191. Yeh, T. F. et al. Intratracheal administration of budesonide/surfactant to prevent bronchopulmonary dysplasia. Am. J. Respir. Crit. Care Med. 193, 86-95 (2016).

192. Roberts, J. K. et al. Pharmacokinetics of budesonide administered with surfactant in premature lambs: implications for neonatal clinical trials. Curr. Clin. Pharmacol. 11, 53-61 (2016).

193. Bland, R. D Albertine K. H Carlton, D. P. \& MacRitchie, A. J. Inhaled nitric oxide effects on lung structure and function in chronically ventilated preterm lambs. Am. J. Respir. Crit. Care Med. 172 899-906 (2005)

194. Cotton, R. B. et al. Inhaled nitric oxide attenuates hyperoxic lung injury in lambs. Pediatr. Res. $\mathbf{5 9}$ 142-146 (2006).

195. Askie, L. M. et al. Inhaled nitric oxide in preterm infants: an individual-patient data meta-analysis of randomized trials. Pediatrics 128, 729-739 (2011)

196. Askie, L. M. et al. Race effects of inhaled nitric oxide in preterm infants: an individual participant data metaanalysis. J. Pediatr. 193, 34-39.e2 (2018).

197. Hwang, S. S., Burris, H. H., Collins, J. W. Jr, Kirpalani, H. $\&$ Wright, C. J. Moving beyond race and ethnicity to understand the effect of inhaled nitric oxide on bronchopulmonary dysplasia prevention. J. Pediatr. 201, 298-300 (2018).

198. Askie, L. M. et al. Association between oxygen saturation targeting and death or disability in extremely preterm infants in the neonatal oxygenation prospective meta-analysis collaboration. JAMA 319 2190-2201 (2018)

199. Collaco, J. M. \& McGrath-Morrow, S. A. Respiratory phenotypes for preterm infants, children, and adults: bronchopulmonary dysplasia and more. Ann. Am. Thorac. Soc. 15, 530-538 (2018).

200. Abman, S. H. \& Nelin, L. D. in The Newborn Lung: Neonatology Questions and Controversies 2nd edn (ed. Bancalari, E.) 407-425 (Elsevier Saunders, 2012)

201. Guaman, M. C. et al. Point prevalence, clinical characteristics, and treatment variation for infants with severe bronchopulmonary dysplasia. Am. J. Perinatol. 32, 960-967 (2015).

202. Gien, J. et al. Retrospective analysis of an interdisciplinary ventilator care program intervention on survival of infants with ventilator-dependent bronchopulmonary dysplasia. Am. J. Perinatol. 34 155-163 (2017).

203. Baker, C. D. et al. A standardized discharge process decreases length of stay for ventilator-dependent children. Pediatrics 137, e20150637 (2016).

204. Burchert, H. \& Lewandowski, A. J. Preterm birth is a novel, independent risk factor for altered cardiac remodeling and early heart failure: is it time for a new cardiomyopathy? Curr. Treat Options Cardiovasc. Med. 21, 8 (2019)

205. Goss, K. N. et al. Early pulmonary vascular disease in young adults born preterm. Am. J. Respiratory Crit. Care Med. 198, 1549-1558 (2018). This is the first study to demonstrate the persistence or resurgence of PVD in young adults who were born preterm and highlights the need for early monitoring of PVD during childhood and throughout adulthood.

206. Laurie, S. S. et al. Exaggerated increase in pulmonary artery pressure during exercise in adults born preterm. Am. J. Respir. Crit. Care Med. 197. 821-823 (2018)

207. Lewandowski, A. J. et al. Preterm heart in adult life: cardiovascular magnetic resonance reveals distinct differences in left ventricular mass, geometry, and function. Circulation 127, 197-206 (2013).

208. Lewandowski, A. J. et al. Right ventricular systolic dysfunction in young adults born preterm. Circulation 128, 713-720 (2013).

209. Maron, B. A. \& Abman, S. H. Translational advances in the field of pulmonary hypertension. Focusing on developmental origins and disease inception for the prevention of pulmonary hypertension. Am. J. Respir. Crit. Care Med. 195, 292-301 (2017).

210. Zivanovic, S. et al. Pulmonary artery pressures in school-age children born prematurely. J. Pediatr. 191 42-49.e3 (2017).

211. Cotten, C. M. et al. Prolonged hospital stay for extremely premature infants: risk factors, center differences, and the impact of mortality on selecting a best-performing center. J. Perinatol. 25, 650-655 (2005).

212. Katz-Salamon, M., Gerner, E. M., Jonsson, B. $\overline{8}$ Lagercrantz, H. Early motor and mental development in very preterm infants with chronic lung disease. Arch. Dis. Child Fetal Neonatal. Ed. 83, F1-F6 (2000).

213. McAleese, K. A., Knapp, M. A. \& Rhodes, T. T. Financial and emotional cost of bronchopulmonary dysplasia. Clin. Pediatr. 32, 393-400 (1993)

214. Gough, A., Spence, D., Linden, M., Halliday, H. L. \& McGarvey, L. General and respiratory health outcomes in adult survivors of bronchopulmonary dysplasia: a systematic review. Chest 141, 1554-1567 (2012).

215. Singer, L. T. et al. Maternal psychological distress and parenting stress after the birth of a very low-birthweight infant. JAMA 281, 799-805 (1999).

216. Lau, R. et al. Parent preferences regarding home oxygen use for infants with bronchopulmonary dysplasia. J. Pediatr. 213, 30-37 (2019).

217. Brady, J. M., Zhang, H., Kirpalani, H. \& DeMauro, S. B. Living with severe bronchopulmonary dysplasia parental views of their child's quality of life. J. Pediatr. 207, 117-122 (2019).

218. Degl, J., Discenza, D. \& Sorrells, K. Remembering the power of stories in pediatric research. J. Pediatr. 207 14-17 (2019).

219. Resch, B., Kurath-Koller, S., Eibisberger, M. \& Zenz, W. Prematurity and the burden of influenza and respiratory syncytial virus disease. World J. Pediatr. 12, 8-18 (2016)

220. Smith, V. C. et al. Rehospitalization in the first year of life among infants with bronchopulmonary dysplasia. J. Pediatr. 144, 799-803 (2004).

221. Townsi, N., Laing, I. A., Hall, G. L. \& Simpson, S. J. The impact of respiratory viruses on lung health after preterm birth. Eur. Clin. Respir. J. 5, 1487214 (2018)

222. Stein, R. T. et al. Respiratory syncytial virus hospitalization and mortality: systematic review and meta-analysis. Pediatr. Pulmonol. 52, 556-569 (2017).

223. Miller, E. K. et al. Human rhinoviruses in severe respiratory disease in very low birth weight infants. Pediatrics 129, e60-e67 (2012).

224. Kotecha, S. J. et al. Effect of preterm birth on later FEV 1: a systematic review and meta-analysis. Thorax 68, 760-766 (2013)

225. Lombardi, E. et al. Lung function in a cohort of 5-yearold children born very preterm. Pediatr. Pulmonol. $\mathbf{5 3}$ 1633-1639 (2018)

226. Sanchez-Solis, M., Perez-Fernandez, V., BoschGimenez, V., Quesada, J. J. \& Garcia-Marcos, L. Lung function gain in preterm infants with and without bronchopulmonary dysplasia. Pediatr. Pulmonol. 51 936-942 (2016)

227. Bobolea, I., Arismendi, E., Valero, A. \& Agusti, A Early life origins of asthma: a review of potential effectors. J. Investig. Allergol. Clin. Immunol. 29 168-179 (2019)

228. MacLean, J. E. et al. Altered breathing mechanics and ventilatory response during exercise in children born extremely preterm. Thorax 71, 1012-1019 (2016).

229. Skromme, K., Leversen, K. T., Eide, G. E., Markestad, T. \& Halvorsen, T. Respiratory illness contributed significantly to morbidity in children born extremely premature or with extremely low birthweights in 1999-2000. Acta Paediatr. 104, 1189-1198 (2015).

230. Vom Hove, M., Prenzel, F., Uhlig, H. H. \& Robel-Tillig, E. Pulmonary outcome in former preterm, very low birth weight children with bronchopulmonary dysplasia: a case-control follow-up at school age. J. Pediatr. 164 40-45.e4 (2014).

231. Joshi, S. et al. Exercise-induced bronchoconstriction in school-aged children who had chronic lung disease in infancy. J. Pediatr. 162, 813-818.e1 (2013).

232. O'Dea, C. A. et al. Increased prevalence of expiratory flow limitation during exercise in children with bronchopulmonary dysplasia. ERJ Open Res. 4 00048-2018 (2018).

233. Balinotti, J. E. et al. Growth of lung parenchyma in infants and toddlers with chronic lung disease of infancy. Am. J. Respir. Crit. Care Med. 181, 1093-1097 (2010).

234. Narayanan, M. et al. Catch-up alveolarization in ex-preterm children: evidence from ${ }^{3} \mathrm{He}$ magnetic resonance. Am. J. Respir. Crit. Care Med. 187, 1104-1109 (2013)

235. Thunqvist, P. et al. Lung function after extremely preterm birth-A population-based cohort study (EXPRESS). Pediatr. Pulmonol. 53, 64-72 (2018)

236. Hirata, K. et al. Longitudinal impairment of lung function in school-age children with extremely low birth weights. Pediatr. Pulmonol. 52, 779-786 (2017).

237. Kennedy, J. D. Lung function outcome in children of premature birth. J. Paediatr. Child Health 35 , 516-521 (1999)

238. Ronkainen, E. et al. New BPD predicts lung function at school age: follow-up study and meta-analysis. Pediatr. Pulmonol. 50, 1090-1098 (2015).

239. Simpson, S. J. et al. Altered lung structure and function in mid-childhood survivors of very preterm birth. Thorax 72, 702-711 (2017). 
240. Urs, R., Kotecha, S., Hall, G. L. \& Simpson, S. J. Persistent and progressive long-term lung disease in survivors of preterm birth. Paediatr. Respir. Rev. 28 87-94 (2018)

241. Simpson, S. J. et al. Lung function trajectories throughout childhood in survivors of very preterm birth: a longitudinal cohort study. Lancet Child Adolesc. Health 2, 350-359 (2018). This study found that 4-12-year-old survivors of preterm birth with BPD are more likely to have impaired lung function trajectories, ongoing respiratory symptoms and abnormal chest CT findings compared with children born at term, indicating that BPD may increase the risk of chronic respiratory disease in later life.

242. Bui, D. S. et al. Childhood predictors of lung function trajectories and future COPD risk: a prospective cohort study from the first to the sixth decade of life. Lancet Respir. Med. 6, 535-544 (2018).

243. Hadchouel, A. et al. Identification of SPOCK2 as a susceptibility gene for bronchopulmonary dysplasia. Am. J. Respir. Crit. Care Med. 184, 1164-1170 (2011)

244. Mullen, M. P. et al. Quality of life and parental adjustment in pediatric pulmonary hypertension. Chest 145, 237-244 (2014).

245. Handler, S. S. et al. Assessment of quality of life in pediatric patients with pulmonary hypertension. Pulm. Circ. 9, 2045894018822985 (2019).

246. Kadmon, G. et al. Pulmonary hypertension specific treatment in infants with bronchopulmonary dysplasia. Pediatr. Pulmonol. 52, 77-83 (2017).

247. Joshi, S. et al. Cardiovascular function in children who had chronic lung disease of prematurity. Arch. Dis. Child Fetal Neonatal Ed. 99, F373-F379 (2014).

248. Koroglu, O. A., Yalaz, M., Levent, E., Akisu, M. \& Kultursay, N. Cardiovascular consequences of bronchopulmonary dysplasia in prematurely born preschool children. Neonatology 104, 283-289 (2013).

249. Levy, P. T., Patel, M. D., Choudhry, S., Hamvas, A. \& Singh, G. K. Evidence of echocardiographic markers of pulmonary vascular disease in asymptomatic infants born preterm at one year of age. J. Pediatr. 197, 48-56.e2 (2018)

250. Assad, T. R. et al. Prognostic effect and longitudinal hemodynamic assessment of borderline pulmonary hypertension. JAMA Cardiol. 2, 1361-1368 (2017)

251. Maron, B. A. et al. Association of borderline pulmonary hypertension with mortality and hospitalization in a large patient cohort: insights from the Veterans Affairs Clinical Assessment, Reporting, and Tracking Program. Circulation 133, 1240-1248 (2016)

252. Douschan, P. et al. Mild elevation of pulmonary arterial pressure as a predictor of mortality. $\mathrm{Am}$. $\mathrm{J}$. Respir. Crit. Care Med. 197, 509-516 (2018).

253. Heresi, G. A et al. Clinical characterization and survival of patients with borderline elevation in pulmonary artery pressure. Pulm. Circ. 3, 916-925 (2013)

254. Horbar, J. D. et al. Variation in performance of neonatal intensive care units in the United States. JAMA Pediatr. 171, e164396 (2017)

255. Soll, R. F. et al. Obstetric and neonatal care practices for infants 501 to $1500 \mathrm{~g}$ from 2000 to 2009 . Pediatrics 132, 222-228 (2013).

256. Cools, F., Offringa, M. \& Askie, L. M. Elective high frequency oscillatory ventilation versus conventional ventilation for acute pulmonary dysfunction in preterm infants. Cochrane Database Syst. Rev. 3, CD000104 (2015).

257. Klingenberg, C., Wheeler, K. I., McCallion, N., Morley, C. J. \& Davis, P. G. Volume-targeted versus pressure-limited ventilation in neonates. Cochrane Database Syst. Rev. 10, CD003666 (2017).

258. Rojas-Reyes, M. X., Morley, C. J. \& Soll, R. Prophylactic versus selective use of surfactant in preventing morbidity and mortality in preterm infants. Cochrane Database Syst. Rev. 3, CD000510 (2012).
259. Doyle, L. W., Cheong, J. L., Ehrenkranz, R. A. \& Halliday, H. L. Early ( $<8$ days) systemic postnatal corticosteroids for prevention of bronchopulmonary dysplasia in preterm infants. Cochrane Database Syst. Rev. 10, CD001146 (2017).

260. Doyle, L. W., Cheong, J. L., Ehrenkranz, R. A. \& Halliday, H. L. Late ( $>7$ days) systemic postnatal corticosteroids for prevention of bronchopulmonary dysplasia in preterm infants. Cochrane Database Syst Rev. 10, CD001145 (2017).

261. Darlow, B. A., Graham, P. J. \& Rojas-Reyes, M. X Vitamin A supplementation to prevent mortality and short- and long-term morbidity in very low birth weight infants. Cochrane Database Syst. Rev. 8 CD000501 (2016)

262. Barrington, K. J., Finer, N. \& Pennaforte, T. Inhaled nitric oxide for respiratory failure in preterm infants. Cochrane Database Syst. Rev. 1, CD000509 (2017).

263. Picarillo, A. P. \& Carlo, W. Using quality improvement tools to reduce chronic lung disease. Clin. Perinatol. 44, 701-712 (2017).

264. Aslam, M. et al. Bone marrow stromal cells attenuate lung injury in a murine model of neonatal chronic lung disease. Am. J. Respir. Crit. Care Med. 180 $1122-1130$ (2009).

265. van Haaften, T. et al. Airway delivery of mesenchymal stem cells prevents arrested alveolar growth in neonatal lung injury in rats. Am. J. Respir. Crit. Care Med. 180, 1131-1142 (2009).

266. Augustine, S. et al. Mesenchymal stromal cell therapy in bronchopulmonary dysplasia: systematic review and meta-analysis of preclinical studies. Stem Cell Transl. Med. 6, 2079-2093 (2017)

267. Chang, Y. S. et al. Mesenchymal stem cells for bronchopulmonary dysplasia: phase 1 dose-escalation clinical trial. J. Pediatr. 164, 966-972.e6 (2014). This is the first phase I trial showing the feasibility and no short-term toxicity of a single intratracheal administration of allogeneic cord-blood-derived mesenchymal stromal cells in extreme preterm infants at risk of developing BPD

268. Powell, S. B. \& Silvestri, J. M. Safety of intratracheal administration of human umbilical cord blood derived mesenchymal stromal cells in extremely low birth weight preterm infants. J. Pediatr. 210, 209-213.e2 (2019).

269. Alvarez-Fuente, M. et al. Off-label mesenchymal stromal cell treatment in two infants with severe bronchopulmonary dysplasia: clinical course and biomarkers profile. Cytotherapy 20, 1337-1344 (2018).

270. Lim, R. et al. First-in-human administration of allogeneic amnion cells in premature infants with bronchopulmonary dysplasia: a safety study. Stem Cell Transl. Med. 7, 628-635 (2018).

271. Fung, M. E. \& Thebaud, B. Stem cell-based therapy for neonatal lung disease: it is in the juice. Pediatr. Res. 75, 2-7 (2014).

272. Lesage, F. \& Thebaud, B. Nanotherapies for micropreemies: stem cells and the secretome in bronchopulmonary dysplasia. Semin. Perinatol. 42 453-458 (2018)

273. Willis, G. R. et al. Mesenchymal stromal cell exosomes ameliorate experimental bronchopulmonary dysplasia and restore lung function through macrophage immunomodulation. Am. J. Respir. Crit. Care Med. 197, 104-116 (2018).

274. Collins, J. J. P. et al. Impaired angiogenic supportive capacity and altered gene expression profile of resident $C D 146^{+}$mesenchymal stromal cells isolated from hyperoxia-injured neonatal rat lungs. Stem Cell Dev. 27, 1109-1124 (2018).

275. Hennrick, K. T. et al. Lung cells from neonates show a mesenchymal stem cell phenotype. Am. J. Respir. Crit. Care Med. 175, 1158-1164 (2007).

276. Mobius, M. A. et al. Oxygen disrupts human fetal lung mesenchymal cells: implications for bronchopulmonary dysplasia. Am. J. Respir. Cell Mol. Biol. 60, 592-600 (2019).
277. Mobius, M. A. \& Thebaud, B. Bronchopulmonary dysplasia - where have all the stem cells gone? Origin and (potential) function of resident lung stem cells. Chest 152, 1043-1052 (2017).

278. Lipsitz, Y. Y., Timmins, N. E. \& Zandstra, P. W. Quality cell therapy manufacturing by design. Nat. Biotechnol. 34, 393-400 (2016)

279. Collins, J. J. P., Tibboel, D., de Kleer, I. M., Reiss, I. K. M. $\&$ Rottier, R. J. The future of bronchopulmonary dysplasia: emerging pathophysiological concepts and potential new avenues of treatment. Front. Med. 4, 61 (2017).

280. Ley, D. et al. rhIGF-1/rhIGFBP-3 in preterm infants: a phase 2 randomized controlled trial. J. Pediatr. 206 56-65.e8 (2019).

281. Treutlein, B. et al. Reconstructing lineage hierarchies of the distal lung epithelium using single-cell RNA-seq. Nature 509, 371-375 (2014).

282. Frank, D. B. et al. Early lineage specification defines alveolar epithelial ontogeny in the murine lung. Proc. Natl Acad. Sci. USA 116, 4362-4371 (2019).

283. Zacharias, W. J. et al. Regeneration of the lung alveolus by an evolutionarily conserved epithelial progenitor. Nature 555, 251-255 (2018)

284. Contreras, M. et al. Bronchoalveolar oxyradical inflammatory elements herald bronchopulmonary dysplasia. Crit. Care Med. 24, 29-37 (1996).

285. Gladstone, I. M. \& Levine, R. L. Oxidation of proteins in neonatal lungs. Pediatrics 93, 764-768 (1994)

286. Thompson, A. \& Bhandari, V. Pulmonary biomarkers of bronchopulmonary dysplasia. Biomark. Insights 3, 361-373 (2008).

287. Whitsett, J. A. \& Alenghat, T. Respiratory epithelial cells orchestrate pulmonary innate immunity. Nat. Immunol. 16, 27-35 (2015).

288. Whitsett, J. A., Wert, S. E. \& Trapnell, B. C. Genetic disorders influencing lung formation and function at birth. Hum. Mol. Genet. 13, R207-R215 (2004).

\section{Acknowledgements}

This work was supported by National Heart, Lung and Blood Institute of the US National Institutes of Health $(\mathrm{NIH})$ grants (U01HL1 22642 and U01HL134745 to J.A.W.; RO1 HL68702, R01HL145679 and U01HL12118-01 to S.H.A.; and K24 HL143283 to M.L.), and funding from the Australian National Health and Medical Research Council (NHMRC) to P. .D and from the Canadian Institute for Health Research, Stem Cell Network and the Ontario Institute for Regenerative Medicine to B.T. The authors thank J. Kitzmiller and C.-L. Na for their contributions in obtaining images.

\section{Author contributions}

Introduction (B.T.); Epidemiology (M.L. and B.T.); Mechanisms/pathophysiology (J.A.W., S.H.A., A.H.J. and B.T); Diagnosis, screening and prevention (R.H.S. J.L.A. and B.T.); Management (P.G.D., S.H.A. and B.T.); Quality of life (K.N.G., S.A.McG.-M. and B.T.); Outlook (R.F.S. and B.T.); Overview of the Primer (B.T.)

\section{Competing interests}

A.H.J. consults occasionally for Chiesi Farmaceutici about BPD and surfactant. S.H.A has served as a consultant for Takeda Pharmaceuticals. R.H.S. is a consultant for Takeda Pharmaceutical Company and for Actelion Pharmaceutical Ltd. The remaining authors declare no competing interests.

\section{Peer review information}

Nature Reviews Disease Primers thanks Y.S. Chang, M. Hallman and the other, anonymous, reviewer(s) for their contribution to the peer review of this work.

\section{Publisher's note}

Springer Nature remains neutral with regard to jurisdictional claims in published maps and institutional affiliations.

RELATED LINKS

NICHD NRN risk estimator: https://neonatal.rti.org/index.cfm 